\title{
DYNAMICAL LOCALIZATION FOR A MULTI-PARTICLE MODEL WITH AN ALLOY-TYPE EXTERNAL RANDOM POTENTIAL
}

\author{
VICTOR CHULAEVSKY ${ }^{1}$, ANNE BOUTET DE MONVEL ${ }^{2}$, AND YURI SUHOV ${ }^{3}$
}

Abstract. We establish lower-edge spectral and dynamical localization for a multiparticle Anderson model in a Euclidean space $\mathbb{R}^{d}, d \geq 1$, in presence of a non-trivial short-range interaction and an alloy-type random external potential.

\section{Introduction. The $N$-particle Hamiltonian in the Continuum}

1.1. The model. This paper follows, with some stylistic modifications (and with a shortened form of presentation), a series of arXiv accounts of an earlier vedrsion of the same work: cf. [6], 7] 9]. We consider an $N$-particle Anderson model in $\mathbb{R}^{d}$ with interaction, where $N>1$ and $d \geq 1$ are arbitrary integers. The model Hamiltonian $\mathbf{H}=\mathbf{H}^{(N)}(\omega)$ is a random Schrödinger operator of the form

$$
\mathbf{H}^{(N)}(\omega)=-\frac{1}{2} \boldsymbol{\Delta}+\mathbf{U}+\mathbf{V}(\omega)
$$

acting on functions from $L^{2}\left(\mathbb{R}^{d} \times \cdots \times \mathbb{R}^{d}\right) \simeq L^{2}\left(\mathbb{R}^{d}\right)^{\otimes N}$. This means that we consider $N$ quantum particles in $\mathbb{R}^{d}$. The joint position vector is $\mathbf{x}=\left(x_{1}, \ldots, x_{N}\right) \in \mathbb{R}^{N d}$, where component $x_{j}=\left(\mathrm{x}_{j}^{(1)}, \ldots, \mathrm{x}_{j}^{(d)}\right) \in \mathbb{R}^{d}$ represents the $j$-th particle, $j=1, \ldots, N$. Next,

$$
-\frac{1}{2} \Delta=-\frac{1}{2} \sum_{1 \leq j \leq N} \Delta_{j}
$$

is the standard kinetic energy operator obtained by adding up the kinetic energies $-\frac{1}{2} \Delta_{j}$ of the individual particles; here, $\Delta_{j}$ denotes the $d$-dimensional Laplacian.

The interaction energy operator is denoted by $\mathbf{U}$ : it is the operator of multiplication by a function $\mathbb{R}^{N d} \ni \mathbf{x} \mapsto U(\mathbf{x})$, the inter-particle potential. Finally, $\mathbf{V}(\omega)$ is the operator of multiplication by a function

$$
\mathbb{R}^{N d} \ni \mathbf{x}=\left(x_{1}, \ldots, x_{N}\right) \longmapsto V\left(x_{1} ; \omega\right)+\cdots+V\left(x_{N} ; \omega\right),
$$

where $V: \mathbb{R}^{d} \times \Omega \rightarrow \mathbb{R}$ is a random field, relative to a probability space $(\Omega, \mathcal{F}, \mathbb{P})$, generating an external potential acting on individual particles.

Assumptions on $U(\mathbf{x})$ and $V(x ; \omega)$ are discussed below. In essence, $U$ is required to be of short-range while $V$ is assumed to be of the so-called alloy-type.

In this paper, we analyse spectral properties of $\mathbf{H}$ by using the method called the Multi-scale analysis (MSA), more precisely, a multi-particle adaptation of a singleparticle 'continuous-space' version of the MSA (MPMSA for short). Our main result is Theorem 1.1] asserting that

Date: October 15, 2010. 
- with probability one the spectrum of operator $\mathbf{H}(\omega)$, in an energy band $I^{0}\left(\eta^{*}\right)=$ $\left[E^{0}, E^{0}+\eta^{*}\right]$ near the lower edge $E^{0}$, is pure point, with an exponential decay of the corresponding eigenfunctions; such a phenomenon is known as exponential spectral localization;

- compactly supported wavepackets $\psi$ remain rapidly decaying (localised) under the action of the reduced propagator $e^{-i t \mathbf{H}} P_{I^{0}\left(\eta^{*}\right)}(\mathbf{H})$, where $P_{I^{0}\left(\eta^{*}\right)}(\mathbf{H})$ is the spectral projection on $I^{0}\left(\eta^{*}\right)$; this phenomenon is called dynamical localization.

Theorem 1.1 is the first rigorous result available in the literature on localization in multi-particle continuous-space Anderson models. For $N=1$ (a single-particle model), spectral localization was initially established in [1], 18 by means of the single-particle MSA (briefly: SPMSA), whereas in 2] dynamical and spectral localization was proved with the help of an alternative Fractional Moment Method (FMM). The fact that dynamical localization is derived in this paper from the MPMSA estimate, in our view puts both forms of localization on essentially equal footage. We thank Abel Klein for the stimulating discussion of this issue.

For lattice (tight-binding) Anderson models, the MPMSA has been developed in papers [12, 13, 14]. An alternative approach based on a multi-particle adaptation of the FMM was successfully employed in [3], still for multi-particle lattice Anderson models; see also 4. Note that the multi-particle version of the FMM, like its singleparticle counterpart, leads directly to the proof of dynamical localization.

1.2. Basic notation. Throughout this paper, we fix integers $N>1$ and $d \geq 1$ (as was said, they can be arbitrary) and work with configurations of $n$ distinguishable quantum particles in $\mathbb{R}^{d}$ where $n$ varies from 1 to $N$. The configuration space of an $n$-particle system is the Euclidean space $\left(\mathbb{R}^{d}\right)^{n}$ which is canonically identified with $\mathbb{R}^{n d}$. A similar identification is used for cubic lattices: $\left(\mathbb{Z}^{d}\right)^{n} \cong \mathbb{Z}^{n d}$.

We use the notation $[[a, b]]$ for segments of integer values: $[[a, b]]:=[a, b] \cap \mathbb{Z}$.

It is convenient to endow $\mathbb{R}^{d}$ and $\mathbb{R}^{n d}$ with max-norms: for $x=\left(\mathrm{x}^{(1)}, \ldots, \mathrm{x}^{(d)}\right) \in \mathbb{R}^{d}$ and $\mathbf{x}=\left(x_{1}, \ldots, x_{N}\right) \in \mathbb{R}^{N d}$,

$$
|x|=\max _{1 \leq i \leq d}\left|\mathrm{x}^{(i)}\right|, \quad|\mathbf{x}|=\max _{1 \leq j \leq n}\left|x_{j}\right| .
$$

The distance 'dist' below is induced by this norm. In terms of the max-norm, the balls of radius $L$ are cubes of sidelength $2 L$ with the edges parallel to the co-ordinate axes (such cubes are alternatively called boxes throughout the paper). Specifically, in $\mathbb{R}^{d}$ the open ball of radius $L$ centered at $u=\left(\mathrm{u}^{(1)}, \ldots, \mathrm{u}^{(d)}\right)$ is

$$
\mathrm{C}_{L}(u):=\underset{i=1}{\stackrel{d}{\times}}\left(\mathrm{u}^{(i)}-L, \mathrm{u}^{(i)}+L\right) \subset \mathbb{R}^{d}
$$

while the ball in $\mathbb{R}^{n d}$ centered at $\mathbf{u}=\left(u_{1}, \ldots, u_{n}\right)$ is

$$
\mathbf{C}_{L}(\mathbf{u})=\underset{j=1}{\stackrel{n}{\times}} \mathrm{C}_{L}\left(u_{j}\right) \subset \mathbb{R}^{n d}
$$

Sometimes we will also use the symbol $\mathbf{C}_{L}^{(n)}(\mathbf{u})$ and use the term an $n$-particle box, to put an emphasis on the number of particles in the system. For our purposes, it suffices to consider only cubes centered at lattice points $u \in \mathbb{Z}^{d}$ and $\mathbf{u} \in \mathbb{Z}^{\text {nd }}$; consequently, letters $u, v, w$ and $\mathbf{u}, \mathbf{v}, \mathbf{w}$ will refer to points in the corresponding lattices.

Given an integer $n \geq 1$, we define for each $j \in[[1, n]]$ the projection $\Pi_{j}: \mathbb{R}^{n d} \rightarrow \mathbb{R}^{d}$ extracting the position of the $j$ th particle: $\Pi_{j}\left(x_{1}, \ldots, x_{n}\right):=x_{j}$. Further, define the 
'full one-particle projection' $\Pi \mathbf{C}_{L}^{(n)}(\mathbf{u})$ of $\mathbf{C}_{L}^{(n)}(\mathbf{u})$ :

$$
\Pi \mathbf{C}_{L}^{(n)}(\mathbf{u}):=\bigcup_{j=1}^{n} \Pi_{j} \mathbf{C}_{L}^{(n)}(\mathbf{u}) \subset \mathbb{R}^{d} .
$$

Given a cube $\mathbf{C}_{L}(\mathbf{u})$, define its outer layer (of width 2), by

$$
\mathbf{C}_{L}^{\text {out }}(\mathbf{u})=\mathbf{C}_{L}(\mathbf{u}) \backslash \mathbf{C}_{L-2}(\mathbf{u}), \quad \mathbf{u} \in \mathbb{Z}^{n d} .
$$

We denote by $\mathbf{1}_{\mathbf{A}}$ the characteristic function of a set $\mathbf{A} \subset \mathbb{R}^{n d}$ and also, with a standard abuse of notation, the operator of multiplication by this function.

From now on we will omit subscript $L_{2}\left(\mathbb{R}^{n d}\right)$ in the notation $\|\cdot\|_{L_{2}\left(\mathbb{R}^{n d}\right)}$ for the vector and operator norms in $L_{2}\left(\mathbb{R}^{n d}\right)$. We will denote by $\mathbf{X}$ the operator of multiplication by the norm

$$
(\mathbf{X} \Psi)(\mathbf{x})=|\mathbf{x}| \Psi(\mathbf{x}), \quad \mathbf{x} \in \mathbb{R}^{N d} .
$$

1.3. External random potential. The random potential acting on each individual particle is of the so-called alloy-type, i.e. it has the following structure:

$$
V(x ; \omega)=\sum_{y \in \mathbb{Z}^{d}} \mathrm{~V}_{y}(\omega) \varphi(x-y),
$$

where the random amplitudes $\mathrm{V}_{y}(\omega)$ satisfy the conditions (E2)-(E3) and the 'bump function' $\varphi$ satisfies the conditions (E4)-(E5); see below. However, our results remain valid in a more general situation where the bump functions are not necessarily identical, provided that conditions (E4)-(E5) hold uniformly.

1.4. Interaction potential. As was said above, the interaction energy operator $\mathbf{U}(\mathbf{x})$ in Eqn (1.1) acts as multiplication by a function $\mathbf{x} \in \mathbb{R}^{N d} \mapsto U(\mathbf{x}) \in \mathbb{R}$. More generally, we suppose that functions $\mathbf{x} \in \mathbb{R}^{n d} \mapsto U(\mathbf{x}) \in \mathbb{R}$ are given, for $n \in[[1, N]]$, accounting for the energy of $n$-particle 'sub-configurations'; this is stressed by employing the common notation $U(\mathbf{x})$. Consequently, the term 'interaction' will address the whole family of these functions. For $n=1$, function $U(x), x \in \mathbb{R}^{d}$, gives a 'background' one-particle potential. In this paper we do not assume isotropy, symmetry or translation invariance of the interaction. However, the condition of finite range (see (1.9) is essential.

Suppose that a partition of a configuration $\mathbf{x} \in \mathbb{R}^{l d}$ is given, into complementary sub-configurations $\mathbf{x}_{\mathcal{J}}=\left(x_{j}\right)_{j \in \mathcal{J}}$ and $\mathbf{x}_{\mathcal{J}^{c}}=\left(x_{j}\right)_{j \in[[1, n]] \backslash \mathcal{J}}$, with $\varnothing \neq \mathcal{J} \subsetneq[[1, N]]$. Next, define the distance between $\mathbf{x}_{\mathcal{J}}$ and $\mathbf{x}_{\mathcal{J}^{c}}$ :

$$
\operatorname{dist}\left(\mathbf{x}_{\mathcal{J}}, \mathbf{x}_{\mathcal{J}^{c}}\right):=\min \left[\left|x_{i}-x_{j}\right|: i \in \mathcal{J}, j \in \mathcal{J}^{\mathrm{c}}\right] .
$$

We say that the interaction has range $\mathrm{r}_{0} \in(0, \infty)$ if, for all $n \in[[2, N]]$ and $\mathbf{x} \in \mathbb{R}^{l d}$,

$$
\operatorname{dist}\left(\mathbf{x}_{\mathcal{J}}, \mathbf{x}_{\mathcal{J}^{c}}\right)>\mathrm{r}_{0} \Longrightarrow U(\mathbf{x})=U\left(\mathbf{x}_{\mathcal{J}}\right)+U\left(\mathbf{x}_{\mathcal{J}^{c}}\right) \text {. }
$$

Observe that the finite range condition does not impose restrictions on one-particle potential $U(x)$ for $x \in \mathbb{R}^{d}$.

\subsection{Assumptions.}

(E1) The interaction has a finite range $\mathrm{r}_{0} \geq 0$ and all functions $x \in \mathbb{R}^{n d} \mapsto U(\mathbf{x})$, $n \in[[1, N]]$, are non-negative and bounded.

(E2) There exists a constant $\mathrm{v} \in(0, \infty)$ such that

$$
\mathbb{P}\left\{0 \leq \mathrm{V}_{0} \leq \mathrm{v}\right\}=1 \text {. }
$$


(E3) The marginal distribution of random variables $\mathrm{V}_{s}$ is uniformly Hölder-continuous: $\exists \mathrm{a}, \mathrm{b}>0$ such that for all $\epsilon \in[0,1]$,

$$
\sup _{t \in \mathbb{R}} \mathbb{P}\left(t \leq \mathrm{V}_{s}<t+\epsilon\right) \leq \mathrm{a} \epsilon^{\mathrm{b}} .
$$

(E4) The function $\varphi: \mathbb{R}^{d} \rightarrow \mathbb{R}$ is nonnegative, bounded and compactly supported:

$$
\operatorname{diam}(\operatorname{supp} \varphi) \leq \mathrm{r}_{1}<\infty \text {. }
$$

(E5) $\forall L \geq 1$ and $u \in \mathbb{Z}^{d}$,

$$
\sum_{y \in \mathrm{C}_{L}(u)} \varphi(x-y) \geq \mathbf{1}_{\mathrm{C}_{L}(u)}(x) .
$$

1.6. The main result. Let $E^{0}$ denote the lower edge of $\mathbf{H}_{0}^{(N)}(\omega)$, where operator $\mathbf{H}_{0}^{(N)}(\omega)$ is given by

$$
\mathbf{H}_{0}^{(N)}(\omega)=-\frac{1}{2} \boldsymbol{\Delta}+\sum_{j=1}^{N} U\left(x_{j}\right)
$$

and defined uniquely as the self-adjoint extension from the set $C_{0}^{2}\left(\mathbf{R}^{N d}\right)$ of compactly supported twice-differentiable functions on $\mathbf{R}^{N d}$. Due to nonnegativity of summands $U\left(x_{j}\right)$, the value $E^{0}$ is nonnegative.

Theorem 1.1. Assume conditions (E1)-(E5) and let operator $\mathbf{H}^{(N)}(\omega)$ be as in Eqn (1.1) (defined formally as a unique self-adjoint extension from the domain $C_{0}^{2}\left(\mathbf{R}^{N d}\right)$ ). Then $\exists$ nonrandom constants $\eta^{*}>0$ and $m^{*}>0$ such that, with $\mathbb{P}$-probability one, either the spectrum of operator $\mathbf{H}^{(N)}(\omega)$ in the interval $I^{0}\left(\eta^{*}\right):=\left[E^{0}, E^{0}+\eta^{*}\right]$ is empty or the following localization properties hold true.

(i) The spectrum of $\mathbf{H}^{(N)}(\omega)$ in $I^{0}\left(\eta^{*}\right)$ is pure point, and the eigenfunctions $\mathbf{\Psi}_{j}(\mathbf{x} ; \omega)$ of $\mathbf{H}^{(N)}(\omega)$ with eigenvalues $E_{j}(\omega) \in I^{0}\left(\eta^{*}\right)$ satisfy exponential bounds

$$
\left\|\mathbf{1}_{\mathbf{C}(\mathbf{u})} \mathbf{\Psi}_{j}(\cdot ; \omega)\right\| \leq c_{j}(\omega) \mathrm{e}^{-m^{*}|\mathbf{u}|}, \quad \mathbf{u} \in \mathbb{Z}^{N d} .
$$

(ii) $\forall s>0$ and any compact subset $\mathbb{K} \subset \mathbb{R}^{N d}$, there exists a number $C=C(s, \mathbb{K}) \in$ $(0,+\infty)$ such that the following bound holds:

$$
\mathbb{E}\left[\sup _{t \in \mathbb{R}}\left\|\mathbf{X}^{s} \mathrm{e}^{-\mathrm{i} t \mathbf{H}^{(N)}(\omega)} \mathbf{P}_{I^{0}\left(\eta^{*}\right)}\left(\mathbf{H}^{(N)}(\omega)\right) \mathbf{1}_{\mathbf{K}}\right\|\right] \leq C,
$$

where $\mathbf{P}_{I^{0}\left(\eta^{*}\right)}\left(\mathbf{H}^{(N)}(\omega)\right)$ stands for the spectral projection of the operator $\mathbf{H}^{(N)}(\omega)$ to $I^{0}\left(\eta^{*}\right)$.

Remarks. 1. In assumption (E1), we can relax the boundedness condition by allowing 'hard-core interactions': $\exists \mathrm{d}_{0} \in\left(0, \mathrm{r}_{0}\right)$ such that $\forall n \in[[2, N]]$ and $\mathbf{x}=$ $\left(x_{1}, \ldots, x_{n}\right) \in \mathbb{R}^{n d}$,

$$
\min _{1 \leq i<j \leq n}\left|x_{i}-x_{j}\right|<\mathrm{d}_{0} \Longrightarrow U(\mathbf{x})=+\infty,
$$

and $U(\mathbf{x})$ is uniformly bounded from above when $\min _{1 \leq i<j \leq n}\left|x_{i}-x_{j}\right| \geq \mathrm{d}_{0}$. 
2. The possibility that the spectrum of $\mathbf{H}$ in $I^{0}\left(\eta^{*}\right)$ is empty can be excluded by more specific conditions in assumption (E1) and (E2). Viz., if, in addition to (1.10), we suppose that $\forall \epsilon>0$ the probability $\mathbb{P}\left(0<\mathrm{V}_{s}<\epsilon\right)>0$ then with $\mathbb{P}$-probability one, the spectrum of operator $\mathbf{H}$ in $I^{0}\left(\eta^{*}\right)$ is non-empty. Hence, by Theorem 1.1 under this additional assumption, the spectrum of $\mathbf{H}$ in $I^{0}\left(\eta^{*}\right)$ is pure point, and the corresponding eigenfunctions are exponentially localized.

If, furthermore, we set $U(x) \equiv 0$ (no background potential) then the value $E^{0}$ becomes 0 .

3. In assumption (E3), Hölder continuity can be relaxed to a form of log-Hölder continuity.

4. A direct application of general results on local regularity of (generalized) eigenfunctions of Schrödinger operators, (cf. Theorem 2.4 from [10; see also 11, 19]) leads to the following property. The eigenfunctions $\boldsymbol{\Psi}_{j}(\mathbf{x} ; \omega)$ with eigenvalues $E^{0} \leq E_{j}(\omega) \leq$ $E^{0}+\eta^{*}$ satisfy the bounds:

$$
\left|\Psi_{j}(\mathbf{x} ; \omega)\right| \leq \widetilde{c}_{j}(\omega) \mathrm{e}^{-\widetilde{m}^{*}|\mathbf{x}|}, \quad \mathbf{x} \in \mathbb{R}^{d \times N}
$$

with $\widetilde{m}^{*}>0$ and random constants $\widetilde{c}_{j}(\omega) \in(0,+\infty)$.

5. Theorem 1.1 addresses the spectrum of operator $\mathbf{H}$ in the whole Hilbert space $L_{2}\left(\mathbb{R}^{N d}\right)$. This, of course, covers subspaces $L_{2}^{\text {sym }}\left(\mathbb{R}^{N d}\right)$ and $L_{2}^{\text {asym }}\left(\mathbb{R}^{N d}\right)$ formed by symmetric and antisymmetric functions (bosonic and fermionic subspaces, respectively).

6. As can be seen from bounds used in the proof of Theorem 1.1 when we increase the number of particles $N$, keeping fixed the assumptions on $U(\mathbf{x})$ and $V(x, \omega)$ and preserving the upper bound on $U(\mathbf{x})$ and the range of interaction $\mathrm{r}_{0}$, the width $\eta^{*}$ guaranteed in Theorem 1.1 tends to 0 (in fact, rather rapidly, which is somewhat disappointing). This can be formally attributed to insufficiencies of the version of MPMSA developed in the current paper, but in fact the roots of the prooblem are deeper and stem from the situation occurring already in the SPMSA (when the dimension $d$ increases). We refer the reader to [7] and [9] for more details.

\section{The MPMSA estimate And SPECTRAL LOCALization}

2.1. Hamiltonians in finite cubes. In the proof of Theorem 1.1 we will focus on properties of finite-volume versions $\mathbf{H}_{\mathbf{C}}=\mathbf{H}_{\mathbf{C}}^{(N)}(\omega)$ of Hamiltonian $\mathbf{H}$. More precisely, let $\mathbf{C}=\mathbf{C}^{(N)}(\mathbf{u})$ be an $N$-particle box and consider the operator $\mathbf{H}_{\mathbf{C}}^{(N)}(\omega)$ in $L^{2}(\mathbf{C})$, (referred to as the Hamiltonian of the $N$-particle system in $\mathbf{C}$ ), of the same structure as in Eqn (1.1):

$$
\mathbf{H}_{\mathbf{C}}^{(N)}(\omega)=-\frac{1}{2} \Delta_{\mathbf{C}}+\mathbf{U}+\mathbf{V}(\omega) .
$$

Here $\boldsymbol{\Delta}_{\mathbf{C}}$ stands for the Laplacian in $\mathbf{C}$ with Dirichlet's boundary conditions on $\partial \mathbf{C}$.

The spectrum of $\mathbf{H}_{\mathbf{C}}$ will be denoted by $\sigma\left(\mathbf{H}_{\mathbf{C}}\right)$.

Under conditions (E1) $-\left(\right.$ E5), for for $\mathbb{P}$-almost all $\omega \in \Omega$, operator $\mathbf{H}_{\mathbf{C}}$ is correctly defined in $L_{2}(\mathbf{C})$, as a self-adjoint extension from the domain $C_{0}^{2}(\mathbf{C})$. Moreover, $\mathbf{H}_{\mathbf{C}}$ has a discrete spectrum, since its resolvent

$$
\mathbf{G}_{\mathbf{C}}(E)=\left(\mathbf{H}_{\mathbf{C}}-E\right)^{-1}, \text { for } E \in \mathbb{R} \backslash \sigma\left(\mathbf{H}_{\mathbf{C}}\right),
$$


is a compact integral operator; properties of $\mathbf{G}_{\mathbf{C}}(E)$ will be at the centre of our attention. The integral kernel

$$
\mathbf{C} \times \mathbf{C} \ni\left(\mathbf{x}, \mathbf{x}^{\prime}\right) \mapsto \mathbf{G}_{\mathbf{C}}\left(\mathbf{x}, \mathbf{x}^{\prime} ; E\right), \quad \mathbf{x}, \mathbf{x}^{\prime} \in \mathbf{C},
$$

is known as the Green function of $\mathbf{H}_{\mathbf{C}}$.

The MPMSA is based on an asymptotical analysis of resolvent $\mathbf{G}_{\mathbf{C}}(E)$ as $\mathbf{C} \nearrow \mathbb{R}^{N d}$. More precisely, cubes $\mathbf{C}$ will have the form

$$
\mathbf{C}=\mathbf{C}_{L_{k}}(\mathbf{u}), \mathbf{u} \in \mathbb{Z}^{N d}, k=0,1, \ldots,
$$

where positive integers $L_{k}$ (called length-scales or briefly scales) are determined by a recurrence involving a starting value $L_{0}$ and a parameter $\alpha>1$ :

$$
L_{k}=\left[L_{k-1}^{\alpha}\right]+1 \sim\left(L_{0}\right)^{\alpha^{k}}, k \geq 1 .
$$

Here [.] stands for the integer part. In future, we will take $\alpha=3 / 2$. Nevertheless, to keep a connection with earlier references, we will continue using symbol $\alpha$. A similar agreement will be applied to the parameter $\beta>0$ appearing below (see Eqn (3.1)): we will set $\beta=1 / 2$. The integer $L_{0}>0$ will be eventually assumed to be sufficiently large.

Summarising, for future references,

$$
\alpha=3 / 2, \quad \beta=1 / 2, \quad L_{0} \text { is a positive integer, large enough. }
$$

Consequently, in the course of the argument, we will often work with $n$-particle Hamiltonians $\mathbf{H}_{\mathbf{C}}^{(n)}=\mathbf{H}_{\mathbf{C}}^{(n)}(\omega)$, of the same form as in (2.1), with $n=1, \ldots, N$. Mutatis mutandis, definitions and facts introduced/noted for an $N$ particle system will be used for a system of $n$ particles as well.

For a concise summary of the MPMSA as an inductive process in $n$ and $L_{k}$ we refer the reader to [13, [14; in the present paper we follow the same principal steps (modified by the transition from a lattice to a continuous-space model). Specifically, to derive the crucial MPMSA estimate (2.14) for the Hamiltonian $\mathbf{H}_{\mathbf{C}_{L_{k}}}^{(N)}$, we assume that these estimates are established for Hamiltonians $\mathbf{H}_{\mathbf{C}_{L_{l}}}^{(n)}$ (i) $\forall n \in[[1, N-1]]$ and length scale $L_{l}, l \geq 0$, and (ii) for $n=N$ and $L_{l}$ with $0 \leq l \leq k-1$; we then aim to reproduce this estimate for $n=N$, at scale $L_{k}$. The basic tools in the case $n=1$ are provided by the SPMSA (cf. the monograph [20] where the continuous-space version of the SPMSA is presented in detail, along with an extensive bibliography). Next, the scale induction for operators $\mathbf{H}_{\mathbf{C}_{L_{k}}}^{(n)}$ with $n>1$ includes two principal components:

- initial $L_{0}$-scale MPMSA bounds;

- derivation of the bounds at scale $L_{k}$, assuming that that they are established at the scale $L_{k-1}$.

These components require distinctive arguments and use different properties from assumptions (E1)- (E5).

2.2. Separable cubes and Wegner-type bounds. As was commented on in 7 ] and 9] (and earlier in [12, 13, 14] and [8 in the context of tight-binding models), the principal difficulty encountered while attempting to extend the SPMSA to the MPMSA is that for $N>1$ the random field $\mathbf{x} \in \mathbb{R}^{N d} \mapsto \sum_{i=1}^{N} V\left(x_{i} ; \omega\right)$ features a 'strong dependence' at distance even when the underlying potential $x \in \mathbb{R}^{d} \mapsto V(x ; \omega)$ is 
generated by IID variables $\mathrm{V}_{y}(\omega)$. To this end, in paper [5] we introduced the concept of separable pairs of multi-particle cubes for which we establish the so-called Wegnertype bounds. For reader's convenience, we reproduce the result of [5] in this subsection.

Definition 2.1. Let $n=1, \ldots, N$ and assume $\mathcal{J}$ is a non-empty subset in $\{1, \ldots, n\}$. We say that a cube $\mathbf{C}_{L}^{(n)}(\mathbf{y})$ is $\mathcal{J}$-separable from cube $\mathbf{C}_{L}^{(n)}(\mathbf{x})$ if

$$
\left(\bigcup_{j \in \mathcal{J}} \Pi_{j} \mathbf{C}_{L+\mathrm{r}_{1}}^{(n)}(\mathbf{y})\right) \bigcap\left(\bigcup_{i \notin \mathcal{J}} \Pi_{i} \mathbf{C}_{L+\mathrm{r}_{1}}^{(n)}(\mathbf{y}) \bigcup \Pi \mathbf{C}_{L+\mathrm{r}_{1}}^{(n)}(\mathbf{x})\right)=\varnothing,
$$

where $R$ is the constant from condition (E3).

Next, a pair of cubes $\mathbf{C}_{L}^{(n)}(\mathbf{x}), \mathbf{C}_{L}^{(n)}(\mathbf{y})$ is said to be separable if $\operatorname{dist}\left(\mathbf{C}_{L}^{(n)}(\mathbf{x}), \mathbf{C}_{L}^{(n)}(\mathbf{y})\right)>$ $2 N\left(L+\mathrm{r}_{1}\right)$ and, for some non-empty set $\mathcal{J} \subset[[1, n]]$,

- either $\mathbf{C}_{L}^{(n)}(\mathbf{y})$ is $\mathcal{J}$-separable from $\mathbf{C}_{L}^{(n)}(\mathbf{x})$,

- or $\mathbf{C}_{L}^{(n)}(\mathbf{x})$ is $\mathcal{J}$-separable from $\mathbf{C}_{L}^{(n)}(\mathbf{y})$.

Fix an interval $I \subset \mathbb{R}$. Given $n=1, \ldots, N, q>0$ and $L_{0} \geq 2$, define the following property $\mathbf{W} \mathbf{2}(n)\left(=\mathbf{W} \mathbf{2}\left(n, L_{0}, \beta\right)\right)$, for random $n$-particle Hamiltonians $\mathbf{H}_{\mathbf{C}}^{(n)}$ where $\mathbf{C}=$ $\mathbf{C}_{l}^{(n)}(\mathbf{x})$ and $l \geq L_{0}$.

$\mathbf{W 2}(n)$ : For all $l \geq L_{0}$ and any separable pair of cubes $\mathbf{C}_{\ell}^{(n)}(\mathbf{x}), \mathbf{C}_{\ell}^{(n)}(\mathbf{y})$,

$$
\mathbb{P}\left\{\exists E \in I \text { : neither } \mathbf{C}_{l}^{(n)}(\mathbf{x}) \text { nor } \mathbf{C}_{l}^{(n)}(\mathbf{y}) \text { is } E \text {-CNR }\right\}<e^{-l^{\beta / 2}} .
$$

Theorem 2.1. For any $q>0$ and a bounded interval $I \subset \mathbb{R}$, there exists $L_{\mathrm{W}}^{*}=$ $L_{\mathrm{W}}^{*}(q, I) \in(0,+\infty)$ such that $\mathbf{W} \mathbf{2}(n)$ holds true for all $n=1, \ldots, N$ and $L_{0} \geq L_{\mathrm{W}}^{*}$.

Proof. See [5].

The following Lemma 2.1 and Corollary 2.1 give useful insight into the property of separability. For the proofs, see [7].

Lemma 2.1. Given $n \geq 2$, set $\kappa(n)=n^{n}$.

(A) For any $L>1$ and $n$-particle configuration $\mathbf{x} \in \mathbb{Z}^{\text {nd }}$, there exists a collection of $n$-particle cubes $\mathbf{C}_{L^{(l)}}\left(\mathbf{x}^{(l)}\right), l=1, \ldots, K(\mathbf{x}, n)$, with $K(\mathbf{x}, n) \leq \kappa(n)$ and $L^{(l)} \leq$ $2 n\left(L+\mathrm{r}_{1}\right)$, such that if a vector $\mathbf{y} \in \mathbb{Z}^{n d}$ satisfies

$$
\mathbf{y} \notin \bigcup_{l=1}^{K(\mathbf{x}, n)} \mathbf{C}_{L^{(l)}}\left(\mathbf{x}^{(l)}\right),
$$

and dist $\left(\mathbf{C}_{L}^{(n)}(\mathbf{x}), \mathbf{C}_{L}^{(n)}(\mathbf{y})\right)>2 n\left(L+\mathrm{r}_{1}\right)$, then the pair $\left(\mathbf{C}_{L}^{(n)}(\mathbf{x}), \mathbf{C}_{L}^{(n)}(\mathbf{y})\right)$ is separable.

(B) A pair of cubes of the form $\mathbf{C}_{L}^{(n)}(\mathbf{x}), \mathbf{C}_{L}^{(n)}(\mathbf{0})$ is separable, provided that

$$
|\mathbf{x}|>2(n+2)\left(L+\mathrm{r}_{1}\right) \text {. }
$$

Corollary 2.1. Fix two integers, $n \geq 2$ and $L>1$, and let $\kappa(n)<\infty$ be the number defined in Lemma 2.1. Set $B=4 n\left(L+\mathrm{r}_{1}\right)+1$ and consider an $n$-particle cube $\mathbf{C}_{L}(\mathbf{x})$ and $2 \kappa(n)+1$ disjoint concentric annular sets $\boldsymbol{A}_{1}(\mathbf{x}), \ldots, \boldsymbol{A}_{2 \kappa(n)+1}$ around $\mathbf{C}_{L}(\mathbf{x})$ :

$$
\boldsymbol{A}_{j}(\mathbf{x})=\mathbf{C}_{L+j B}(\mathbf{x}) \backslash \mathbf{C}_{L+(j-1) B}(\mathbf{x}), \quad j=1, \ldots, 2 \kappa(n)+1 .
$$

Then at least one of the annuli $\boldsymbol{A}_{2 j-1}(\mathbf{x}), 1 \leq j \leq \kappa(n)+1$, contains no cube $\mathbf{C}_{L}(\mathbf{y})$ not separable from $\mathbf{C}_{L}(\mathbf{x})$. 
2.3. Singularity and double singularity. The crucial bound in the MPMSA is an upper-bound for the probability of the so-called double singularity (2.14). It is based on the following concept.

Definition $2.2((E, m)$-singularity and nonsingularity). Let $E \in \mathbb{R}$ and $m>0$. We say that cube $\mathbf{C}_{L}^{(n)}(\mathbf{u}) \subset \mathbb{R}^{n d}, 1 \leq n \leq N$, is $(E, m)$-nonsingular $((E, m)$-NS) if $E \in \mathbb{R} \backslash \sigma\left(\mathbf{H}_{\mathbf{C}_{L}(\mathbf{u})}\right)$ and for any $\mathbf{y} \in \mathbf{C}_{L}(\mathbf{u})_{L}^{\text {out }}(\mathbf{u}) \cap \mathbb{Z}^{N d}$, the $L_{2}$-norm of the operator $\mathbf{1}_{\mathbf{C}_{1}(\mathbf{u})} \mathbf{G}_{\mathbf{C}_{L}(\mathbf{u})}(E) \mathbf{1}_{\mathbf{C}_{1}(\mathbf{y})}$ satisfies the bound

$$
\left\|\mathbf{1}_{\mathbf{C}_{1}(\mathbf{u})} \mathbf{G}_{\mathbf{C}_{L}(\mathbf{u})}(E) \mathbf{1}_{\mathbf{C}_{1}(\mathbf{y})}\right\| \leq \mathrm{e}^{-\gamma(m, L, n)},
$$

where the quantity $\gamma(m, L, n)\left(=\gamma_{N}(m, L, n)\right)$ is defined by

$$
\gamma(m, L, n):=m L\left(1+L^{-1 / 8}\right)^{N-n+1}, \quad 1 \leq n \leq N .
$$

Otherwise, $\mathbf{C}_{L}(\mathbf{u})$ is called (E,m)-singular $((E, m)-S)$.

Observe that $\mathrm{e}^{-\gamma(m, L, n)}<\mathrm{e}^{-m L}$, for any $L>0$ and $n \in[1, N]$.

Given an integer $N>2$ and numbers $p_{0}, b>0$, introduce a sequence of positive numbers,

$$
P\left(n, k, p_{0}\right)=p_{0}(1+b)^{k} 4^{N-n}, n=1, \ldots, N .
$$

The only restriction upon the parameter $p_{0}$ is that

$$
p_{0}>N d \alpha .
$$

For notational brevity, the argument $p_{0}$ of the function $P$ will sometimes be omitted, unless its value is to be specified explicitly.

The value of parameter $b$ can be specified as $b=1 / 3$ but the only place where it matters is Eqn (4.4). Therefore, we will keep symbol $b$ to make the algebraic manipulations more transparent.

Further, given an interval $I \subset \mathbb{R}$, numbers $m>0, n \in[[1, N]]$ and $k \geq 0$, consider the following property.

$\operatorname{DS}\left(m, p_{0}, k, I, n\right)$ : For all separable pairs of cubes $\mathbf{C}_{L_{k}}^{(n)}(\mathbf{u}), \mathbf{C}_{L_{k}}^{(n)}(\mathbf{v})$

$$
\mathbb{P}\left\{\exists E \in I: \mathbf{C}_{L_{k}}^{(n)}(\mathbf{u}) \text { and } \mathbf{C}_{L_{k}}^{(n)}(\mathbf{v}) \text { are }(E, m)-\mathrm{S}\right\} \leq L_{k}^{-2 P\left(n, k, p_{0}\right)} .
$$

Recall: $L_{k}$ stands for an integer of the form (2.4), with $\alpha=3 / 2$ as in 2.5. The abbreviation DS means 'double singularity'.

As was mentioned before, property $\mathbf{D S}\left(m, p_{0}, k, I, N\right)$ (which addresses Hamiltonians $\mathbf{H}_{\mathbf{C}_{L_{k}}(\mathbf{u})}^{(n)}(\omega)$ and $\left.\mathbf{H}_{\mathbf{C}_{L_{k}}(\mathbf{v})}^{(n)}(\omega)\right)$, is critical for the $N$-particle MSA scheme; see Theorem 2.2 below, establishing connections with the spectrum of the Hamiltonian $\mathbf{H}^{(N)}(\omega)$. Once this property is verified for all $k \geq 0$ (at the end of Section 6), it will mark the end of the proof of assertion (i) of Theorem 1.1.

Theorem 2.2. Let $I \subset \mathbb{R}$ be an interval. Assume that for some $m>0, L_{0}>2$, $p_{0}>N d \alpha$ and for any $k \geq 0$, property $\mathbf{D S}\left(m, p_{0}, k, I, N\right)$ holds true, with $L_{k}$ as in Eqns (2.4), (2.5).

Then, with $\mathbb{P}$-probability one,

(i) The spectrum of operator $\mathbf{H}^{(N)}(\omega)$ in I is pure point. 
(ii) The eigenfunctions $\mathbf{\Psi}_{j}(\mathbf{x} ; \omega)$ of $\mathbf{H}^{(N)}(\omega)$ with eigenvalues $E_{j}(\omega) \in I$ satisfy the exponential bounds similar to Eqn (1.15):

$$
\left\|\mathbf{1}_{\mathbf{C}(\mathbf{u})} \mathbf{\Psi}_{j}(\cdot ; \omega)\right\| \leq c_{j}(\omega) \mathrm{e}^{-m|\mathbf{u}|}, \quad \mathbf{u} \in \mathbb{Z}^{N d} .
$$

Theorem 2.2 represents an 'analytic' part of the MPMSA. (Probability plays a subordinate role here, reduced merely to the Borel-Cantelli lemma, which is guaranteed by the fact that $p>N d \alpha$.) The proof of Theorem 2.2 follows the same line of arguments as in previous publications and is omitted; cf. 13, [14]. (In fact, the idea of the proof has not changed since [15.)

In view of Theorem [2.2 assertion (i) of Theorem 1.1] can be deduced from the following Theorem 2.3

Theorem 2.3. Under assumptions of Theorem 1.1, there exist $\eta^{*}>0$ sufficiently small, $p^{*}>N d \alpha$, and $m^{*}>0$ such that, for an integer $L_{0}>1$ large enough, property $\mathbf{D S}\left(m, p_{0}, k, I, N\right)$ holds for all $k \geq 0$, with $p_{0}=p^{*}, m=m^{*}$, interval $I$ of the form $I^{0}\left(\eta^{*}\right)=\left[E^{0}, E^{0}+\eta^{*}\right]$ and length-scale $L_{k}$ as in Eqns (2.4), (2.5).

Sections 3 [6] are devoted to the proof of Theorem [2.3 This theorem represents a 'probabilistic' part of the MPMSA; unlike Theorem 2.2 its proof is quite sensitive to particulars of a given model. Nevertheless, we will follow the same logical scheme as in [14, Theorem 3].

Assertion (ii) of Theorem 1.1 is established in Section 7

\section{The $N$-PARTiCle MSA induCtion SCheme}

In view of Theorem 2.2 our aim is to check property $\mathbf{D S}\left(m, p_{0}, k, I, n\right)$, i.e., (2.14), for $n=N$. As was mentioned before, it is done by means of a combined induction, in both $k$ and $n$. Consequently, in some definitions below we refer to the particle number parameter $n$, whereas in other definitions - where we want to stress the passage from $N-1$ to $N$ - we will use the capital letter.

The reader may assume from the start that the interval $I$ is of the form $I^{0}\left(\eta^{*}\right)$.

3.1. The initial scale. The initial step of the MPMSA induction consists in establishing properties $\mathbf{D S}\left(m, p_{0}, k, I, n\right)$, for $k=0$ and for $n=1, \ldots, N$.

Lemma 3.1. Fix any $p_{0}>0$ and integers $N, d \geq 1$. There exists $\eta_{0}^{*}>0$ such that $\mathbf{D S}\left(m, p_{0}, 0, I, n\right)$ holds true for all $n \in[[1, N]]$ with the interval $I^{0}\left(\eta^{*}\right)$.

The assertion of Lemma 3.1 follows directly from [20. Theorems 2.2.3, 3.3.3] and is omitted from the paper. It is instructive to observe that the proofs in [20] do not rely upon a single-particle structure of the potential and can be easily adapted to multi-particle Hamiltonians.

3.2. The scaling step inequality. Lemma 3.2 below is based on a construction alternative to [15. Section 4, Lemma 4.2] but uses the same basic idea and serves the same purpose. The proof can be found in [7].

Definition 3.1 ( $E$-complete non-resonance). Given $E \in I$ and $\mathbf{v} \in \mathbb{Z}^{n d}$, the $n$-particle cube $\mathbf{C}_{L}(\mathbf{v})$ is called

(i) E-nonresonant (E-NR) if

$$
\operatorname{dist}\left(E, \sigma\left(\mathbf{H}_{\mathbf{C}_{L}(\mathbf{v})}\right)\right) \geq \mathrm{e}^{-L^{\beta}}
$$

and $E$-resonant $(E-\mathrm{R})$ if the opposite inequality holds; 
(ii) E-completely non-resonant $(E-\mathrm{CNR})$ if $\mathbf{C}_{L}(\mathbf{v})$ is $E$-NR and does not contain any $E$-resonant cube $\mathbf{C}_{\ell}^{(n)}(\mathbf{w})$ with $\ell \geq L^{1 / \alpha}$.

Definition 3.2 (Discretized Green function). Given cubes $\mathbf{C}=\mathbf{C}_{L}(\mathbf{u})$, value $E \in$ $\mathbb{R} \backslash \sigma\left(\mathbf{H}_{\mathbf{C}}\right)$ and vectors $\mathbf{v}, \mathbf{w} \in \mathbf{B}:=\mathbf{C} \cap \mathbb{Z}^{N d}$, we now denote

$$
\mathrm{D}_{L, \mathbf{u}}(\mathbf{v}, \mathbf{w} ; E)=\left\|\mathbf{1}_{\mathbf{C}_{1}(\mathbf{v})} \mathbf{G}_{\mathbf{C}}(E) \mathbf{1}_{\mathbf{C}_{1}(\mathbf{w})}\right\| .
$$

We call function $\mathbf{B} \times \mathbf{B} \ni(\mathbf{v}, \mathbf{w}) \mapsto \mathrm{D}_{L, \mathbf{u}}(\mathbf{v}, \mathbf{w} ; E)$ the discretized Green function for $\mathbf{H}_{\mathbf{C}}$. The same definition is applicable for $\mathbf{C}_{\ell}(\mathbf{u})$ yielding a function

$$
\mathbf{B}_{\ell}(\mathbf{u}) \times \mathbf{B}_{\ell}(\mathbf{u}) \ni(\mathbf{v}, \mathbf{w}) \mapsto \mathrm{D}_{\ell, \mathbf{u}}(\mathbf{v}, \mathbf{w} ; E) \text {, where } \mathbf{B}_{\ell}(\mathbf{u}):=\mathbf{C}_{\ell}(\mathbf{u}) \cap \mathbb{Z}^{N d} .
$$

Lemma 3.2. Given $n=1, \ldots, N, m>0, M>0$ and integer $J \geq 1$, consider an $n$-particle box $\mathbf{C}_{L}(\mathbf{u})$. There exists an integer $L_{\mathrm{scal}}^{*}=L_{\mathrm{scal}}^{*}(m, M, J)$ with the following property. Suppose that the conditions $(\mathrm{A})-(\mathrm{C})$ are satisfied:

(A) $\mathbf{C}_{L}(\mathbf{u})$ is E-CNR;

(B) there exists a family $\left\{\mathbf{A}_{i}\right\}$ of at most $J$ disjoint annuli $\mathbf{A}_{i}=\mathbf{C}_{l_{i}+r_{i}}(u) \backslash \mathbf{C}_{l_{i}}(u)$ of total width $\sum r_{i} \leq M L^{1 / \alpha}$ such that any box $\mathbf{C}_{\ell}(\mathbf{v}) \subset \mathbf{C}_{L}(\mathbf{u}) \backslash\left(\cup_{i} \mathbf{A}_{i}\right)$ is NS;

(C) $L \geq L_{\text {scal }}^{*}$.

Then box $\mathbf{C}_{L}(\mathbf{u})$ is NS:

$$
\max _{|\mathbf{x}-\mathbf{u}| \leq L^{1 / \alpha}} \max _{\mathbf{y} \in \mathbf{C}_{L}^{\text {out }}(\mathbf{u}) \cap \mathbb{Z}^{N d}}\left|\mathrm{D}_{\mathbf{C}_{L}(\mathbf{u})}(\mathbf{x}, \mathbf{y} ; E)\right| \leq \mathrm{e}^{-\gamma(m, L, n)} .
$$

3.3. The inductive step. The inductive step of the MPMSA induction consists in deducing, for a given value $k \geq 0$, property $\mathbf{D S}\left(m, p_{0}, k+1, I, N\right)$ from properties $\mathbf{D S}\left(m, p_{0}, k, I, n\right)$ assumed for all $n=1, \ldots, N$ and properties $\mathbf{D S}\left(m, p_{0}, k+1, I, n\right)$ assumed for all $n=1, \ldots, N-1$. Let us summarise:

Theorem 3.1. Fix any integers $N, d \geq 1$. There exist values $L_{+}^{*}>0, \eta_{+}^{*}>0$ and two functions

$$
m:\left(0, \eta^{*}\right] \rightarrow \mathbb{R}_{+}, \quad p_{0}:\left(0, \eta^{*}\right] \rightarrow \mathbb{R}_{+}, \text {with } p_{0}(\eta) \nearrow+\infty \text { as } \eta \rightarrow 0,
$$

with the following property:

Set $I^{0}(\eta)=\left[E^{0}, E^{0}+\eta\right]$ with $\eta \in\left(0, \eta_{0}^{*}\right)$ and suppose that for some $k \geq 0$ and $L_{0} \geq L_{+}^{*}$

- property $\mathbf{D S}\left(m(\eta), p_{0}(\eta), k, I^{0}(\eta), n\right)$ holds for all $n \in[[1, N]]$;

- property $\mathbf{D S}\left(m(\eta), p_{0}(\eta), k+1, I^{0}(\eta), n\right)$ holds for all $n \in[[1, N-1]]$.

Then $\mathbf{D S}\left(m(\eta), p_{0}(\eta), k+1, I^{0}(\eta), N\right)$ also holds.

The rest of the Sections 3 6 is devoted to the proof of Theorem 3.1. Observe that once this proof is completed, Theorem 2.3 and hence assertion (i) Theorem 1.1 will be established.

From now on, parameter $p_{0}$ will be considered as depending on $\eta$, but for simplicity of notation, the argument $\eta$ will be omitted when it does not cause confusion.

3.4. Interactive cubes. The assertion of Theorem 3.1 i.e., the deduction of property $\mathbf{D S}\left(m, p_{0}, k+1, I, N\right)$ from $\mathbf{D S}\left(m, p_{0}, k, I, N\right)$ and $\mathbf{D S}\left(m, p_{0}, k+1, I, n\right)$ with $n=$ $1, \ldots, N-1$ is done separately for the three types of pairs of separable boxes $\mathbf{C}_{L_{k+1}}^{(N)}(\mathbf{u})$, $\mathbf{C}_{L_{k+1}}^{(N)}(\mathbf{v})$, according to their position relative to the 'interaction strip' in $\mathbb{R}^{N d}$. 
Recall: $r_{0} \in(0,+\infty)$ is the interaction radius (cf. (E1)). Consider the following subset in $\mathbb{R}^{n d}, n=1, \ldots, N$ :

$$
\mathbb{D}^{(n)}=\left\{\mathbf{x}=\left(x_{1}, \ldots, x_{n}\right) \in \mathbb{R}^{n d}: \max _{1 \leq j_{1}, j_{2} \leq n}\left|x_{j_{1}}-x_{j_{2}}\right| \leq N \mathrm{r}_{0}\right\}
$$

It is plain that

$$
\forall \mathbf{x} \in \mathbb{R}^{n d} \backslash \mathbb{D}^{(n)} \exists(\varnothing \neq) \mathcal{J} \subsetneq[[1, N]]: \min _{j_{1} \in \mathcal{J}} \min _{j_{2} \notin \mathcal{J}}\left|x_{j_{1}}-x_{j_{2}}\right|>\mathrm{r}_{0}
$$

Definition 3.3 (Interactive cubes). Let $\mathbf{C}_{L}^{(n)}(\mathbf{u})$ be an $n$-particle cube. We say that $\mathbf{C}_{L}^{(n)}(\mathbf{u})$ is fully interactive $(\mathrm{FI})$ when $\mathbf{C}_{L}^{(n)}(\mathbf{u}) \cap \mathbb{D}^{(n)} \neq \varnothing$, and partially interactive (PI), otherwise.

The term 'fully interactive' simply means that the respective $n$-particle system cannot be decomposed into a union of two or more non-interacting subsystems, while such a decomposition is guaranteed for the 'partially interactive' cubes. It will be convenient to fix one such decomposition (referred to as canonical), for each PI box:

$$
\mathbf{C}_{L}^{(N)}(\mathbf{u})=\mathbf{C}_{L}^{\left(n^{\prime}\right)}\left(\mathbf{u}_{\mathcal{J}}\right) \times \mathbf{C}_{L}^{\left(n^{\prime \prime}\right)}\left(\mathbf{u}_{\mathcal{J}^{c}}\right), n^{\prime}=|\mathcal{J}| \geq 1, n^{\prime \prime}=\left|\mathcal{J}^{c}\right| \geq 1
$$

(The actual choice is irrelevant for our arguments.)

The three types of pairs of separable boxes considered below are as follows.

(I) Both cubes $\mathbf{C}_{L_{k+1}}^{(N)}(\mathbf{u}), \mathbf{C}_{L_{k+1}}^{(N)}(\mathbf{v})$ are PI.

(II) Both $\mathbf{C}_{L_{k+1}}^{(N)}(\mathbf{u}), \mathbf{C}_{L_{k+1}}^{(N)}(\mathbf{v})$ are FI.

(III) One of $\mathbf{C}_{L_{k+1}}^{(N)}(\mathbf{u}), \mathbf{C}_{L_{k+1}}^{(N)}(\mathbf{v})$ is FI, while the other is PI.

These three cases are treated in Sections 4 [5 and [6 respectively. The end of proof of Theorem 3.1] is achieved at the end of Section 6

Throughout Sections 3 6, we work with a fixed bounded interval $I \subset \mathbb{R}$ and variable $n=1, \ldots, N$.

We conclude this section by describing one of important technical tools used below.

3.5. Resolvent inequality. Using the so-called Geometric Resolvent Inequality for Schrödinger operators in Euclidean spaces, one can establish the following statement:

(DGRI) Discretized geometric resolvent inequality: Given cubes $\mathbf{C}_{\ell}(\mathbf{v}) \subset \mathbf{C}_{L-3}(\mathbf{u})$,

$$
\begin{aligned}
& \forall \mathbf{y} \in \mathbf{C}_{L}^{\text {out }}(\mathbf{u}) \text { and } E \in I \backslash\left(\sigma\left(\mathbf{H}_{\mathbf{C}_{\ell}(\mathbf{u})}\right) \cup \sigma\left(\mathbf{H}_{\mathbf{C}_{L}(\mathbf{u})}\right)\right), \\
& \mathrm{D}_{L, \mathbf{u}}(\mathbf{v}, \mathbf{y} ; E) \leq C^{(0)} \sum_{\mathbf{w} \in \mathbf{C}_{\ell}^{\text {out }}(\mathbf{v}) \cap \mathbb{Z}^{N d}} \mathrm{D}_{\ell, \mathbf{v}}(\mathbf{v}, \mathbf{w} ; E) \mathrm{D}_{L, \mathbf{u}}(\mathbf{w}, \mathbf{y} ; E) .
\end{aligned}
$$

Our task in the remaining part of the paper will be essentially reduced to the analysis of decay of functions $D_{L_{k}, \mathbf{u}}(\mathbf{v}, \mathbf{w} ; E)$ for $E \in \mathbb{R} \backslash \sigma\left(\mathbf{H}_{\mathbf{C}_{L_{k}}(\mathbf{u})}\right)$, when vectors $\mathbf{v}$ and $\mathbf{w}$ are distant apart (viz., $\mathbf{v}$ is 'deeply' inside $\mathbf{C}_{L_{k}}(\mathbf{u})$ whereas $\mathbf{w}$ is near the boundary of $\mathbf{C}_{L_{k}}(\mathbf{u})$; see below).

\section{Case I: Pairs of Partially interactive singular cubes}

In this section, we will be able to derive property $\mathbf{D S}\left(m, p_{0}, k+1, I, N\right)$ for a pair of PI separable cubes $\mathbf{C}_{L_{k+1}}^{(N)}(\mathbf{u}), \mathbf{C}_{L_{k+1}}^{(N)}(\mathbf{v})$ without referring to $\mathbf{D S}\left(m, p_{0}, k, I, N\right)$. However, we will use properties $\mathbf{D S}\left(m, p_{0}, k+1, I, n\right)$ for $n=1, \ldots, N-1$. 
Let $\mathbf{C}=\mathbf{C}_{L_{k+1}}^{(N)}(\mathbf{u})$ be a PI-cube where $\mathbf{u}=\left(u_{1}, \ldots, u_{N}\right) \in \mathbb{Z}^{N d}$. Let $\mathcal{J} \subset\{1, \ldots, N\}$ be a proper subset figuring in Eqn (3.6). Write $\mathbf{u}=\left(\mathbf{u}^{\prime}, \mathbf{u}^{\prime \prime}\right)$ where $\mathbf{u}^{\prime}=\mathbf{u}_{\mathcal{J}}=$ $\left(u_{j}\right)_{j \in \mathcal{J}} \in\left(\mathbb{Z}^{d}\right)^{\mathcal{J}}$ and $\mathbf{u}^{\prime \prime}=\mathbf{u}_{\mathcal{J}^{c}}=\left(u_{j}\right)_{j \notin \mathcal{J}} \in\left(\mathbb{Z}^{d}\right)^{\mathcal{J}^{c}}$ are the corresponding subconfigurations in $\mathbf{u}$. Let $n^{\prime}=|\mathcal{J}|$ be the cardinality of $\mathcal{J}$ and $n^{\prime \prime}=N-n^{\prime}$. We write the respective cube $\mathbf{C}$ as the Cartesian product

$$
\mathbf{C}=\mathbf{C}^{\prime} \times \mathbf{C}^{\prime \prime}, \text { where } \mathbf{C}^{\prime}=\mathbf{C}_{L_{k+1}}^{\left(n^{\prime}\right)}\left(\mathbf{u}^{\prime}\right), \mathbf{C}^{\prime \prime}=\mathbf{C}_{L_{k+1}}^{\left(n^{\prime \prime}\right)}\left(\mathbf{u}^{\prime \prime}\right) .
$$

The Hamiltonian $\mathbf{H}_{\mathbf{C}_{L_{k+1}}^{(N)}(\mathbf{u})}^{(N)}$ can be represented as

$$
\mathbf{H}_{\mathbf{C}}^{(N)}=\mathbf{H}_{\mathbf{C}^{\prime}}^{\left(n^{\prime}\right)} \otimes \mathbf{I}^{\left(n^{\prime \prime}\right)}+\mathbf{I}^{\left(n^{\prime}\right)} \otimes \mathbf{H}_{\mathbf{C}^{\prime \prime}}^{\left(n^{\prime \prime}\right)} .
$$

Here $\mathbf{I}^{\left(n^{\prime}\right)}$ and $\mathbf{I}^{\left(n^{\prime \prime}\right)}$ are the identity operators on $L_{2}\left(\mathbf{C}^{\prime}\right)$ and $L_{2}\left(\mathbf{C}^{\prime \prime}\right)$, respectively.

Definition 4.1 (( $I, m)$-partial tunneling). Fix a number $m>0$ and an interval $I \subset \mathbb{R}$.

(i) An $n$-particle cube $\mathbf{C}_{L_{k+1}}^{(n)}(\mathbf{u})$, with $n<N$, is $(I, m)$-tunneling $((I, m)$-T) if there exists $E \in I$ and two separable $n$-particle cubes $\mathbf{C}_{L_{k}}^{(n)}\left(\mathbf{v}_{j}\right) \subset \mathbf{C}_{L_{k+1}}^{(n)}(\mathbf{u}), j=1,2$ which are $(E, m)-\mathrm{S}$.

(ii) An $N$-particle PI cube $\mathbf{C}_{L_{k+1}}^{(N)}$ (u) is $(I, m)$-partially tunelling $((I, m)$-PT) if at least one of the cubes $\mathbf{C}_{L_{k+1}}^{\left(n^{\prime}\right)}\left(\mathbf{u}_{\mathcal{J}}\right), \mathbf{C}_{L_{k+1}}^{\left(n^{\prime \prime}\right)}\left(\mathbf{u}_{\mathcal{J}^{c}}\right)$ figuring in its canonical decomposition

$$
\mathbf{C}_{L_{k+1}}^{(N)}(\mathbf{u})=\mathbf{C}_{L_{k+1}}^{\left(n^{\prime}\right)}\left(\mathbf{u}_{\mathcal{J}}\right) \times \mathbf{C}_{L_{k+1}}^{\left(n^{\prime \prime}\right)}\left(\mathbf{u}_{\mathcal{J}^{c}}\right),
$$

is $(I, m)$-tunneling. Otherwise, it is called $(I, m)$-non-tunneling $((I, m)$-NT).

Lemma 4.1 (Cf. Lemma 5.2 from [7]). Fix an interval $I \subset \mathbb{R}$ and consider an $N$ particle cube $\mathbf{C}_{L_{k+1}}^{(N)}(\mathbf{u})$. Assume that $\mathbf{C}_{L_{k+1}}^{(N)}(\mathbf{u})$ is $\mathrm{PI},(I, m)$-NT for some $m>0$, and $E$-CNR for some $E \in I$. Then, for $L_{0}$ large enough, the cube $\mathbf{C}_{L_{k+1}}^{(N)}(\mathbf{u})$ is $(E, m)$-NS.

The proof can be found in [7].

Lemma 4.2. Given $m>0, p_{0}>0$, a bounded interval $I \subset \mathbb{R}$ and $n=1, \ldots, N-1$, suppose that property $\mathbf{D S}\left(m, p_{0}, k+1, I, n\right)$ holds for some $k \geq 0$. Then, for any $\mathbf{u} \in \mathbb{Z}^{n d}$,

$$
\mathbb{P}\left\{\mathbf{C}_{L_{k+1}}^{(n)}(\mathbf{u}) \text { is } m-\mathrm{PT}\right\} \leq \frac{1}{2}\left|\mathbf{C}_{L_{k+1}}^{(n)}(\mathbf{u})\right|^{2} \times L_{k}^{-2 P\left(n, k, p_{0}\right)}=\frac{1}{2} L_{k+1}^{-2 P\left(n, k, p_{0}\right) / \alpha+2 N d} .
$$

Proof. Combine $\mathbf{D S}\left(m, p_{0}, k+1, I, n\right)$ with a straightforward upper bound $\frac{1}{2}\left|\mathbf{C}_{L_{k+1}}^{(n)}(\mathbf{u})\right|^{2}$ for the number of pairs of cubes $\mathbf{C}_{L_{k}}^{(n)}\left(\mathbf{y}_{j}\right) \subset \mathbf{C}_{L_{k+1}}^{(n)}(\mathbf{u}), j=1,2$, centered at lattice points.

Lemma 4.3. Suppose $m>0$ and a bounded interval $I \subset \mathbb{R}$ have been given. Let $\mathbf{C}=\mathbf{C}_{L_{k+1}}^{(N)}(\mathbf{u})$ be an $N$-particle PI-cube. Then for any $p_{0}>N d \alpha$ there exists $\eta_{\mathrm{PT}}^{*} \in$ $(0,+\infty)$ and $b>0$ such that if $\eta \in\left(0, \eta_{\mathrm{PT}}^{*}\right)$, then

$$
\mathbb{P}\{\mathbf{C} \text { is } m-\mathrm{PT}\} \leq \frac{1}{4} L_{k+1}^{-2 p_{0}(1+b)^{k+1}} .
$$

Proof. By Definition 4.1 $\mathbf{C}$ with canonical decomposition $\mathbf{C}=\mathbf{C}^{\prime} \times \mathbf{C}^{\prime \prime}$ of the form (3.7) is $m-\mathrm{PT}$ if and only if at least one of its canonical projections, $\mathbf{C}^{\prime}=\mathbf{C}_{L}^{\left(n^{\prime}\right)}\left(\mathbf{u}_{\mathcal{J}}\right)$ 
or $\mathbf{C}^{\prime \prime}=\mathbf{C}_{L}^{\left(n^{\prime \prime}\right)}\left(\mathbf{u}_{\mathcal{J}^{c}}\right)$, is $m$-T. By Lemma 4.2 Eqn (4.2) holds for both $n=n^{\prime}$ and $n=n^{\prime \prime}$, with the exponent in the RHS of Eqn (4.2) bounded as follows:

$$
\frac{2 P\left(N, k+1, p_{0}\right)}{\alpha}-2 N d \geq \frac{2 p_{0}(1+b)^{k} 4^{N-(N-1)}}{\alpha}-2 N d>2 p_{0}(1+b)^{k+1},
$$

with $b>0$, provided that

$$
p_{0}(1+b)^{k}>p_{0}>\frac{N d \alpha}{4-\alpha} ; \quad b<\frac{4-\alpha}{\alpha}-\frac{N d}{p_{0}} .
$$

The left-hand group of inequalities in Eqn (4.4) follows from the condition $p_{0}>N d \alpha$ while the right-hand group holds by inspection for $b=1 / 3$ and $\alpha=3 / 2$ (again with the help of $\left.p_{0}>N d \alpha\right)$.

Lemma 4.4. Given $L_{0} \geq 1, m>0, q>0, p_{0}>N d \alpha$ and a bounded interval $I \subset \mathbb{R}$, assume that

- the bound (4.3) holds true,

- for all $n=1, \ldots, N-1$ the bound (4.2) holds,

- the bound (2.7) with $n=N$ (i.e., property $\mathbf{W 2}(N)$ ) is satisfied,

- $L_{0}$ is sufficiently large.

Then, for any $k \geq 0$ and any pair of separable, PI cubes $\mathbf{C}_{L_{k}}^{(N)}(\mathbf{u})$ and $\mathbf{C}_{L_{k}}^{(N)}(\mathbf{v})$,

$$
\mathbb{P}\left\{\exists E \in I: \mathbf{C}_{L_{k+1}}^{(N)}(\mathbf{u}), \mathbf{C}_{L_{k+1}}^{(N)}(\mathbf{v}) \text { are }(E, m)-S\right\} \leq \frac{1}{2} L_{k+1}^{-2 p(1+b)^{k}}+e^{-L_{k+1}^{\beta / 2}} .
$$

Proof. Set $\mathbf{C}(\mathbf{u})=\mathbf{C}_{L_{k+1}}^{(N)}(\mathbf{u})$ and $\mathbf{C}(\mathbf{v})=\mathbf{C}_{L_{k+1}}^{(N)}(\mathbf{v})$. Lemma 4.1 implies

$$
\mathbb{P}\{\exists E \in I: \mathbf{C}(\mathbf{u}) \text { and } \mathbf{C}(\mathbf{v}) \text { are }(E, m)-\mathbf{S}\}
$$

$\leq \mathbb{P}\{\mathbf{C}(\mathbf{u})$ or $\mathbf{C}(\mathbf{v})$ is $-\mathrm{PT}\}+\mathbb{P}\{\exists E \in I: \mathbf{C}(\mathbf{u})$ and $\mathbf{C}(\mathbf{v})$ are not $E$-CNR $\}$.

The assertion now follows from the assumptions of Lemma 4.4 and from the statement of Lemma 4.3.

Theorem 4.1. Given $p^{*}>N d \alpha$, there exist $m_{\mathrm{PI}}^{*}>0, \eta_{\mathrm{PI}}^{*}>0$ and a positive $L_{\mathrm{PI}}^{*}<$ $+\infty$ with the following property. Take $L_{0} \geq L_{\mathrm{PI}}^{*}$. Then, $\forall k \geq 0$, DS $\left(m, p_{0}, k+1, I, N\right)$ holds for all separable pairs of $N$-particle PI-cubes $\mathbf{C}_{L_{k+1}}^{(N)}(\mathbf{u}), \mathbf{C}_{L_{k+1}}^{(N)}(\mathbf{v})$ with $m=m_{\mathrm{PI}}^{*}$, $p_{0}=p^{*}$ and the interval $I$ of the form $I^{0}\left(\eta_{\mathrm{PI}}^{*}\right)=\left[E^{0}, E^{0}+\eta_{\mathrm{PI}}^{*}\right]$.

Proof. The claim is an immediate corollary of Theorem 2.1 and Lemma 4.4

For future use, consider the following

Definition 4.2. Given an $E$-CNR $N$-particle cube $\mathbf{C}=\mathbf{C}_{L_{k+1}}(\mathbf{u})$, introduce the following quantities:

- $\nu_{\mathrm{PI}}(\mathbf{C} ; E)=$ the maximal number of pairwise separable $(E, m)-\mathrm{S}$ PI cubes $\mathbf{C}_{L_{k}}(\mathbf{x}) \subset \mathbf{C}, \mathbf{x} \in \mathbb{Z}^{N d}$;

- $\widetilde{\nu}_{\mathrm{PI}}(\mathbf{C} ; E)=$ the minimal number of cubes of radius $4 N\left(L+\mathrm{r}_{1}\right)$ needed to cover all PI $(E, m)$-S cubes $\mathbf{C}_{L_{k}}(\mathbf{x}) \subset \mathbf{C}, \mathbf{x} \in \mathbb{Z}^{N d}$;

- $\nu_{\mathrm{FI}}(\mathbf{C} ; E)=$ the maximal number of pairwise separable FI $(E, m)-\mathrm{S}$ cubes $\mathbf{C}_{L_{k}}(\mathbf{x}) \subset \mathbf{C}, \mathbf{x} \in \mathbb{Z}^{N d}$;

- $\nu_{\mathrm{S}}(\mathbf{C} ; E)=$ the minimal number of cubes of radius $4 N\left(L+\mathrm{r}_{1}\right)$ needed to cover all $(E, m)$-S cubes $\mathbf{C}_{L_{k}}(\mathbf{x}) \subset \mathbf{C}, \mathbf{x} \in \mathbb{Z}^{N d}$. 
Clearly,

$$
\nu_{\mathrm{S}}(\mathbf{C} ; E) \leq \widetilde{\nu}_{\mathrm{PI}}(\mathbf{C} ; E)+\nu_{\mathrm{FI}}(\mathbf{C} ; E) .
$$

It is worth noting that there might be several families of pairwise separable cubes in $\mathbf{C}$ (resp., PI pairwise separable, or FI pairwise separable) which are $(E, m)$-S for a given $E$. By $\nu_{\mathrm{PI}}(\mathbf{C} ; E)$ and $\nu_{\mathrm{PI}}(\mathbf{C} ; E)$ we mean the maximal cardinalities of such collections of cubes. These maxima are well-defined since diam $\mathbf{C}<\infty$.

Lemma 4.5. Given $0<\eta<\min \left[\eta_{0}^{*}, \eta_{\mathrm{PI}}^{*}\right], L_{0} \geq 1, q>0, p_{0} \geq N d \alpha$ and a bounded interval $I \subset \mathbb{R}$, assume that

- the bound (4.3) holds true,

- for all $n=1, \ldots, N-1$ the bound (4.2) holds,

- the bound (2.7) with $n=N$ (i.e., property $\mathbf{W 2}(N)$ ) is satisfied,

- $L_{0}$ is sufficiently large.

Then the following inequality takes place:

$$
\mathbb{P}\left\{\exists E \in I: \nu_{\mathrm{PI}}(\mathbf{C} ; E) \geq 2\right\} \leq \frac{1}{2} L_{k}^{2 N d \alpha}\left(L_{k}^{-8 p_{0}(1+b)^{k}}+e^{-L_{k}^{\beta / 2}}\right) .
$$

Proof. If $\nu_{\mathrm{PI}} \geq 2$, then there exist at least two separable singular cubes $\mathbf{C}_{L_{k}}(\mathbf{x})$, $\mathbf{C}_{L_{k}}(\mathbf{y})$. The number of possible pairs $(\mathbf{x}, \mathbf{y})$ is bounded by $\frac{1}{2} L_{k+1}^{2 N d}$, while for a given pair $\mathbf{C}_{L_{k}}(\mathbf{x}), \mathbf{C}_{L_{k}}(\mathbf{y})$ Lemma 4.4 applies. This leads to the assertion of Lemma4.5.

\section{CASe II: Pairs of Fully interactive Singular CUbes}

Recall, the definition of an FI-cube was related to $\mathrm{r}_{0} \in(0,+\infty)$, the range of interaction. Further, the notion of a separable cubes $\mathbf{C}_{L}(\mathbf{u}), \mathbf{C}_{L}(\mathbf{v})$ was related to the constant $r_{1}$, the diameter of support of the bump functions, and included the condition

$$
\operatorname{dist}\left(\mathbf{C}_{L}(\mathbf{u}), \mathbf{C}_{L}(\mathbf{v})\right)>2 N\left(L+\mathrm{r}_{1}\right)
$$

(see Definition 2.1). Before we proceed further, let us state a geometric assertion (see the proof in Appendix A):

Lemma 5.1. Let $L>\mathrm{r}_{0}$ be an integer. Let $\mathbf{C}_{L}\left(\mathbf{u}^{\prime}\right)$ and $\mathbf{C}_{L}\left(\mathbf{u}^{\prime \prime}\right)$ be two separable $N$-particle FI-cubes, where $\mathbf{u}^{\prime}=\left(u_{1}^{\prime}, \ldots, u_{N}^{\prime}\right), \mathbf{u}^{\prime \prime}=\left(u_{1}^{\prime \prime}, \ldots, u_{N}^{\prime \prime}\right)$. Then

$$
\Pi \mathbf{C}_{L+\mathrm{r}_{1}}\left(\mathbf{u}^{\prime}\right) \cap \Pi \mathbf{C}_{L+\mathrm{r}_{1}}\left(\mathbf{u}^{\prime \prime}\right)=\varnothing .
$$

Lemma 5.1 is used in the proof of Lemma 5.2 which, in turn, is a part of the proof of Lemma [5.4 instrumental in establishing Theorem [5.1]

Let an interval $I \subset \mathbb{R}$ and a number $m>0$ be given. Consider the following assertion which is a particular case of $\mathbf{D S}\left(m, p_{0}, k, I, N\right)$ (cf. Eqn 2.14):

$\mathbf{F I S}\left(k, p_{0}, N, I\right)$ : For any pair of separable $N$-particle FI-cubes $\mathbf{C}_{L_{k}}^{(N)}(\mathbf{u})$ and $\mathbf{C}_{L_{k}}^{(N)}(\mathbf{v})$

$$
\mathbb{P}\left\{\exists E \in I: \mathbf{C}_{L_{k}}^{(N)}(\mathbf{u}) \text { and } \mathbf{C}_{L_{k}}^{(N)}(\mathbf{v}) \text { are }(E, m)-\mathrm{S}\right\} \leq L_{k}^{-2 P\left(N, k, p_{0}\right)} .
$$

Lemma 5.2. Let $k \geq 0$ be given. Assume that property $\mathbf{F I S}\left(k, p_{0}, N, I\right)$ holds true. Let $\mathbf{C}=\mathbf{C}_{L_{k+1}}^{(N)}(\mathbf{u})$ be an $N$-particle cube. Then, for any $\ell \geq 1$,

$$
\mathbb{P}\left\{\exists E \in I: \nu_{\mathrm{FI}}(\mathbf{C} ; E) \geq 2 \ell\right\} \leq L_{k}^{2 \ell(2+d \alpha)} \cdot L_{k}^{-2 \ell P\left(N, k, p_{0}\right)} .
$$


The proof can be found in [7]; it is fairly straightforward and based on the independence of the operators $\mathbf{H}_{\mathbf{C}}$ relative to separable FI-cubes.

In the proof of Lemma [5.4 we use the following simple geometric fact which follows from Lemma 2.1

Lemma 5.3. Let $n \in[[1, N]]$ and consider an n-particle cube $\mathbf{C}=\mathbf{C}_{L_{k+1}}(\mathbf{u})$. If, for a given $E \in \mathbb{R}, \nu_{\mathrm{PI}}(\mathbf{C}, E)<2$ then there exist a collection of cubes $\mathbf{C}_{2 N\left(L+\mathrm{r}_{1}\right)}\left(\mathbf{x}^{(i)}\right), i=$ $1, \ldots, J$, where $J \leq \kappa(n)+1$ such that any $\mathrm{PI}$ cube $\mathbf{C}_{L_{k}}(\mathbf{y}) \subset \mathbf{C}_{L_{k+1}}(\mathbf{u})$ disjoint from all $\mathbf{C}_{2 N\left(L_{k}+\mathrm{r}_{1}\right)}\left(\mathbf{x}^{(i)}\right)$ is $(E, m)$-NS. Consequently, $\widetilde{\nu}_{\mathrm{PI}}(\mathbf{C}, E) \leq \kappa(n)+1$. Respectively, if all $(E, m)$-S PI cubes cannot be covered by a family of $\kappa(n)+1$ cubes of radius $2 N\left(L_{k}+\mathrm{r}_{1}\right)$, then $\nu_{\mathrm{PI}}(\mathbf{C}, E) \geq 2$.

Lemma 5.4 (Cf. Lemma 6.3 from [7]). Given $k \geq 0$, let $\mathbf{C}=\mathbf{C}_{L_{k+1}}^{(N)}(\mathbf{u})$ be an $N$ particle cube. Consider a bounded interval $I \subset \mathbb{R}$ and assume that the conditions of Lemma 4.5 are fufilled and property $\mathbf{F I S}\left(k, p_{0}, N, I\right)$ holds true. Then, for any $\ell \geq 1$,

$$
\begin{aligned}
\mathbb{P}\left\{\exists E \in I: \nu_{\mathrm{S}}(\mathbf{C} ; E)>2 \ell+\kappa(N)+1\right\} \\
\quad \leq L_{k}^{2 N d \alpha} \cdot L_{k}^{-2 P\left(N, k, p_{0}\right)}+L_{k}^{2 \ell(2+d \alpha)} \cdot L_{k}^{-2 \ell P\left(N, k, p_{0}\right)} .
\end{aligned}
$$

Proof. Suppose that $\nu_{\mathrm{S}}(\mathbf{C} ; E)>2 \ell+\kappa(N)+1$. Then, owing to Lemma 5.3 either $\nu_{\mathrm{PI}}(\mathbf{C} ; E) \geq 2$ or $\nu_{\mathrm{FI}}(\mathbf{C} ; E) \geq 2 \ell$. Therefore,

$$
\begin{aligned}
\mathbb{P}\{\exists & \left.E \in I: \nu_{\mathrm{S}}(\mathbf{C} ; E)>2 \ell+\kappa(N)+1\right\} \\
& \leq \mathbb{P}\left\{\exists E \in I: \nu_{\mathrm{PI}}(\mathbf{C} ; E) \geq 2\right\}+\mathbb{P}\left\{\exists E \in I: \nu_{\mathrm{FI}}(\mathbf{C} ; E) \geq 2 \ell\right\} \\
& \leq L_{k}^{2 N d \alpha} \cdot L_{k}^{-2 P\left(N, k, p_{0}\right)}+L_{k}^{2 \ell(2+d \alpha)} \cdot L_{k}^{-2 \ell P\left(N, k, p_{0}\right)},
\end{aligned}
$$

by virtue of (4.8) and (5.3).

An elementary calculation gives rise to the following

Corollary 5.1. Under assumptions of Lemma [5.4 with $\ell \geq 2, p_{0}(\eta)$ large enough and for $L_{0}$ large enough, we have, for any integer $k \geq 0$,

$$
\mathbb{P}\left\{\exists E \in I: \nu_{\mathrm{S}}(\mathbf{C} ; E) \geq 2 \ell+2\right\} \leq L_{k+1}^{-2 P\left(N, k, p_{0}\right)-1} .
$$

Now, if two $N$-particle cubes $\mathbf{C}_{L_{k+1}}^{(N)}\left(\mathbf{u}^{\prime}\right)$ and $\mathbf{C}_{L_{k+1}}^{(N)}\left(\mathbf{u}^{\prime \prime}\right)$ are separable, then property W2 $(N)$ (i.e., Eqn (2.7) with $n=N)$ implies the following bound:

$$
\begin{aligned}
\mathbb{P}\left\{\forall E \in I, \text { either } \mathbf{C}_{L_{k+1}}^{(N)}\left(\mathbf{u}^{\prime}\right) \text { or } \mathbf{C}_{L_{k+1}}^{(N)}\left(\mathbf{u}^{\prime \prime}\right) \text { is } E \text {-CNR }\right\} \\
\geq 1-L_{k+1}^{2 N d \alpha} e^{-L_{k}^{\beta^{\prime}}}>1-e^{-L_{k}^{\beta^{\prime}} / 2} .
\end{aligned}
$$

The main result of this section is the following

Theorem 5.1. For any $p^{*}>N d \alpha$, there exist $m_{\mathrm{FI}}^{*}>0, \eta_{\mathrm{FI}}^{*}>0$ and $L_{\mathrm{FI}}^{*} \in(0,+\infty)$ such that the following property holds true. Given $L_{0} \geq L_{\mathrm{FI}}^{*}$ and $k \geq 0$, assume that property $\mathbf{F I S}\left(k, p_{0}, N, I\right)$ holds with $m=m_{\mathrm{FI}}^{*}, p_{0}=p^{*}$ and the interval $I^{0}\left(\eta_{\mathrm{FI}}^{*}\right)=$ $\left[E^{0}, E^{0}+\eta_{\mathrm{FI}}^{*}\right]$. Then property $\mathbf{F I S}\left(k+1, p_{0}, N, I\right)$ also holds, again with $m=m_{\mathrm{FI}}^{*}$, $p_{0}=p^{*}$ and the interval $I=I^{0}\left(\eta_{\mathrm{FI}}^{*}\right)$.

Proof. Let $m>0, \mathbf{u}, \mathbf{v} \in \mathbb{Z}^{N d}$ and assume that $\mathbf{C}_{L_{k+1}}^{(N)}(\mathbf{u})$ and $\mathbf{C}_{L_{k+1}}^{(N)}(\mathbf{v})$ are separable FI-cubes. With an interval $I^{0}(\eta)$ of the form $\left[E^{0}, E^{0}+\eta\right]$, consider the following two 
events:

$$
\begin{aligned}
\mathcal{B} & =\left\{\exists E \in I^{0}(\eta): \mathbf{C}_{L_{k+1}}^{(N)}(\mathbf{u}) \text { and } \mathbf{C}_{L_{k+1}}^{(N)}(\mathbf{v}) \text { are }(E, m)-\mathrm{S}\right\}, \\
\mathcal{D} & =\left\{\exists E \in I: \text { neither } \mathbf{C}_{L_{k+1}}^{(N)}(\mathbf{u}) \text { nor } \mathbf{C}_{L_{k+1}}^{(N)}(\mathbf{v}) \text { is } E \text {-CNR }\right\} .
\end{aligned}
$$

The argument that follows assumes that parameters $m, \eta, p$ and $L_{0}$ are adjusted in the way specified in the conditions of Theorem 5.1] Owing to property W2 $(N)$ (cf. Eqn (2.7), with $n=N$ ), we have: $\mathbb{P}\{\mathcal{D}\}<e^{-L_{k}^{\beta^{\prime}} / 2}$. Moreover, $\mathbb{P}\{\mathcal{B}\} \leq \mathbb{P}\{\mathcal{D}\}+$ $\mathbb{P}\left\{\mathcal{B} \cap \mathcal{D}^{\mathrm{c}}\right\}$. So, it suffices to estimate the probability $\mathbb{P}\left\{\mathcal{B} \cap \mathcal{D}^{\mathrm{c}}\right\}$. Within the event $\mathcal{B} \cap \mathcal{D}^{\mathrm{c}}$, for any $E \in I$, either $\mathbf{C}_{L_{k+1}}^{(N)}(\mathbf{u})$ or $\mathbf{C}_{L_{k+1}}^{(N)}(\mathbf{v})$ must be $E$-CNR. Without loss of generality, assume that for some $E \in I, \mathbf{C}_{L_{k+1}}^{(N)}(\mathbf{u})$ is $E$-CNR and $(E, m)$-S. By Lemma 3.2 if $L_{0}$ (and, therefore, any $L_{k}$ ) is sufficiently large, for such value of $E$, $\nu_{\mathrm{S}}\left(\mathbf{C}_{L_{k+1}}^{(N)}(\mathbf{u}) ; E\right) \geq K+1$, with $K$ as in Lemma 3.2 Now let $K=\kappa(N)$, where $\kappa(N)$ is the constant from Lemma 2.1 We see that

$$
\mathcal{B} \cap \mathcal{D}^{\mathrm{c}} \subset\left\{\exists E \in I: \nu_{\mathrm{S}}\left(\mathbf{C}_{L_{k+1}}^{(N)}(\mathbf{u}) ; E\right) \geq \kappa(N)+1\right\}
$$

and, therefore, by Lemma 5.4 and Corollary 5.1

$$
\mathbb{P}\left\{\mathcal{B} \cap \mathcal{D}^{\mathrm{c}}\right\} \leq \mathbb{P}\left\{\exists E \in I \mid \nu_{\mathrm{S}}\left(\mathbf{C}_{L_{k+1}}^{(N)}(\mathbf{u}) ; E\right) \geq \kappa(N)+1\right\} \leq L_{k}^{-2 P\left(N, k, p_{0}\right)} .
$$

\section{Mixed SeParable Pairs of Singular Cubes}

It remains to derive property $\mathbf{D S}\left(m, p_{0}, k+1, I, N\right)$ in case (III), i.e., for mixed pairs of $N$-particle cubes (where one is FI and the other PI).

A natural counterpart of Theorem 5.1 for mixed pairs of cubes is the following

Theorem 6.1. For any $p^{*}>N d \alpha$, there exist $m_{\mathrm{MI}}^{*}>0, \eta_{\mathrm{MI}}^{*}>0$ and $L_{\mathrm{MI}}^{*} \in(0,+\infty)$ guaranteing the following property. Given $L_{0} \geq L_{\mathrm{FI}}^{*}$ and $k \geq 0$, assume that property $\mathbf{D S}\left(m, p_{0}, k, I, N\right)$ holds, with $m=m_{\mathrm{MI}}^{*}, p_{0}=p^{*}$ and the interval $I$ of the form $I^{0}\left(\eta_{\mathrm{MI}}\right)=\left[E^{0}, E^{0}+\eta_{\mathrm{MI}}^{*}\right]$,

- for any pair of separable PI-cubes $\mathbf{C}_{L_{k}}^{(N)}(\mathbf{x}), \mathbf{C}_{L_{k}}^{(N)}(\mathbf{y}), \mathbf{x}, \mathbf{y} \in \mathbb{Z}^{N d}$,

- for any pair of separable FI-cubes $\mathbf{C}_{L_{k}}^{(N)}(\tilde{\mathbf{x}}), \mathbf{C}_{L_{k}}^{(N)}(\tilde{\mathbf{y}}), \tilde{\mathbf{x}}, \tilde{\mathbf{y}} \in \mathbb{Z}^{N d}$.

Then property $\mathbf{D S}\left(m, p_{0}, k+1, I, N\right)$ holds for all mixed pairs (of separable cubes).

Proof. Assume that $\mathbf{C}_{L_{k+1}}^{(N)}(\mathbf{u}), \mathbf{C}_{L_{k+1}}^{(N)}(\mathbf{v})$ is a separable pair where cube $\mathbf{C}_{L_{k+1}}^{(N)}(\mathbf{u})$ is FI and $\mathbf{C}_{L_{k+1}}^{(N)}(\mathbf{v})$ PI. Consider the following three events:

$$
\begin{aligned}
& \mathcal{B}=\left\{\exists E \in I: \mathbf{C}_{L_{k+1}}^{(N)}(\mathbf{u}) \text { and } \mathbf{C}_{L_{k+1}}^{(N)}(\mathbf{v}) \text { are }(E, m)-\mathrm{S}\right\}, \\
& \mathcal{T}=\left\{\mathbf{C}_{L_{k+1}}(\mathbf{v}) \text { is }(I, m)-\mathrm{PT}\right\}, \\
& \mathcal{D}=\left\{\exists E \in I: \text { neither } \mathbf{C}_{L_{k+1}}^{(N)}(\mathbf{u}) \text { nor } \mathbf{C}_{L_{k+1}}^{(N)}(\mathbf{v}) \text { is } E-\mathrm{CNR}\right\} .
\end{aligned}
$$

By virtue of [4.3),

and by Theorem 2.1

$$
\mathbb{P}\{\mathcal{T}\} \leq \frac{1}{4} L_{k+1}^{-2 P\left(N, k, p_{0}\right)}
$$

$$
\mathbb{P}\{\mathcal{D}\} \leq L_{k+1}^{2 N d} e^{-L_{k+1}^{\beta / 2}} \leq e^{-\frac{1}{2} L_{k+1}^{\beta / 2}},
$$


provided that $L_{0}$ is large enough. Further,

$$
\mathbb{P}\{\mathcal{B}\} \leq \mathbb{P}\{\mathcal{T}\}+\mathbb{P}\left\{\mathcal{B} \cap \mathcal{T}^{\mathrm{c}}\right\} \leq \frac{1}{4} L_{k+1}^{-2 P\left(N, k, p_{0}\right)}+\mathbb{P}\left\{\mathcal{B} \cap \mathcal{T}^{\mathrm{c}}\right\}
$$

Thus, we have

$$
\mathbb{P}\left\{\mathcal{B} \cap \mathcal{T}^{\mathrm{c}}\right\} \leq \mathbb{P}\{\mathcal{D}\}+\mathbb{P}\left\{\mathcal{B} \cap \mathcal{T}^{\mathrm{c}} \cap \mathcal{D}^{\mathrm{c}}\right\} \leq e^{-\frac{1}{2} L_{k+1}^{\beta / 2}}+\mathbb{P}\left\{\mathcal{B} \cap \mathcal{T}^{\mathrm{c}} \cap \mathcal{D}^{\mathrm{c}}\right\} .
$$

Next, within the event $\mathcal{B} \cap \mathcal{T}^{\mathrm{c}} \cap \mathcal{D}^{\mathrm{c}}$, either $\mathbf{C}_{L_{k+1}}^{(N)}(\mathbf{u})$ or $\mathbf{C}_{L_{k+1}}^{(N)}(\mathbf{v})$ is $E$-CNR. It must be the FI-cube $\mathbf{C}_{L_{k+1}}^{(N)}(\mathbf{u})$. Indeed, by Lemma 4.1 had cube $\mathbf{C}_{L_{k+1}}^{(N)}(\mathbf{v})$ been both $E$-CNR and $(I, m)-\mathrm{NT}$, it would have been $(E, m)-\mathrm{NS}$, which is not allowed within the event $\mathcal{B}$. Thus, the cube $\mathbf{C}_{L_{k+1}}^{(N)}(\mathbf{u})$ must be $E$-CNR, but $(E, m)$-S. Hence,

$$
\mathcal{B} \cap \mathcal{T}^{\mathrm{c}} \cap \mathcal{D}^{\mathrm{c}} \subset\left\{\exists E \in I: \mathbf{C}_{L_{k+1}}^{(N)}(\mathbf{u}) \text { is }(E, m) \text {-S and } E \text {-CNR }\right\} .
$$

However, applying Lemma 3.2, we see that

$$
\begin{aligned}
& \left\{\exists E \in I: \mathbf{C}_{L_{k+1}}^{(N)}(\mathbf{u}) \text { is }(E, m)-\mathrm{S} \text { and } E \text {-CNR }\right\} \\
& \quad \subset\left\{\exists E \in I: \nu_{\mathrm{S}}\left(\mathbf{C}_{L_{k+1}}^{(N)}(\mathbf{u}) ; E\right) \geq 2 \ell+\kappa(N)+1\right\} .
\end{aligned}
$$

Therefore,

$$
\begin{aligned}
\mathbb{P}\left\{\mathcal{B} \cap \mathcal{T}^{\mathrm{c}} \cap \mathcal{D}^{\mathrm{c}}\right\} & \leq \mathbb{P}\left\{\exists E \in I: \nu_{\mathrm{S}}\left(\mathbf{C}_{L_{k+1}}^{(N)}(\mathbf{u}) ; E\right) \geq 2 \ell+\kappa(N)+1\right\} \\
& \leq 2 L_{k+1}^{-1} L_{k+1}^{-2 P\left(N, k, p_{0}\right)} .
\end{aligned}
$$

Finally, we get for sufficiently large $L_{0}$ :

$$
\begin{aligned}
\mathbb{P}\{\mathcal{B}\} & \leq \mathbb{P}\{\mathcal{B} \cap \mathcal{T}\}+\mathbb{P}\{\mathcal{D}\}+\mathbb{P}\left\{\mathcal{B} \cap \mathcal{T}^{\mathrm{c}} \cap \mathcal{D}^{\mathrm{c}}\right\} \\
& \leq \frac{1}{2} L_{k+1}^{-2 P\left(N, k, p_{0}\right)}+e^{-\frac{1}{2} L_{k+1}^{\beta / 2}}+2 L_{k+1}^{-1} L_{k+1}^{-2 P\left(N, k, p_{0}\right)} \leq L_{k+1}^{-2 P\left(N, k, p_{0}\right)}
\end{aligned}
$$

This completes the proof of Theorem 6.1

Therefore, Theorem 3.1 and the assertion (i) of Theorem 1.1 are also proven.

\section{From MPMSA bounds to DYNAMiCAL LOCALIZATion}

In this section we establish assertion (ii) of Theorem 1.1 To this end we adapt a well-known scheme used earlier in the single-particle context; cf. the original papers [16]17 and the monograph 20]. Recall again that all cubes appearing in our arguments are centered at lattice points $\mathbf{u} \in \mathbb{Z}^{N d}$; this makes it possible to use some standard combinatorial estimates.

7.1. Step 1. Probability of 'bad' samples. In thsi subsection we assume the property $\mathbf{D S}\left(m, p_{0}, k, I, n\right)$ proven for a given $N \geq 1$ and a given ???bounded??? interval $I \subset \mathbb{R}$ and all $k \geq 0$. ??? Interval $I$ for the time being can be general, but in due course will take form $I^{0}(\eta)$ assumed in Theorem 1.1 ??? Parameter $P\left(N, k, p_{0}\right)$ grows with $k$, therefore for $k$ large enough, we can make the value of $P\left(N, k, p_{0}\right)$ arbitrarily large. Hence, changing if necessary the scales by setting $\widetilde{L}_{k}=L_{k+k_{\odot}}$ with $k_{\circ}$ large enough, we can use the bound $\mathbf{D S}\left(m, p_{0}, k, I, n\right)$ for the new scales $\widetilde{L}_{k}$ and with an arbitrarily large $p_{0}>01$ For notational brevity, we set below $p=P\left(N, 0, p_{0}\right)=p_{0}$. Recall that the assertion (ii) of Theorem 1.1 (see Eqn (1.16)) is claimed for any $s>0$.

\footnotetext{
${ }^{1}$ This is the main place where we benefit from making parameter $P\left(n, k, p_{0}\right)$ growing with $k$.
} 
To prove this fact, we first fix a value $s>0$ for which we want to establish (1.16) and then choose $p$ obeying

$$
2 p>3 N d \alpha^{2}+\alpha s,
$$

changing the scales $L_{k}$ if necessary. Next, for each $j \geq 1$ consider the event

$$
\begin{aligned}
& \mathcal{S}_{j}=\left\{\omega: \exists E \in I \exists \mathbf{y}, \mathbf{z} \in \mathbf{C}_{L_{j+1}}(\mathbf{0})\right. \text { such that } \\
&\left.\mathbf{C}_{L_{j-1}}(\mathbf{y}), \mathbf{C}_{L_{j-1}}(\mathbf{z}) \text { are separable and }(m, E) \text {-singular }\right\} .
\end{aligned}
$$

Further, for $k \geq 1$ denote

$$
\Omega_{k}^{(\mathrm{bad})}=\bigcup_{j \geq k} \mathcal{S}_{j}
$$

Lemma 7.1. $\exists$ a constant $c^{(1)}=c^{(1)}(d, N, p) \in(0,+\infty)$ such that $\forall k \geq 1$ :

$$
\mathbb{P}\left\{\Omega_{k}^{(\mathrm{bad})}\right\} \leq c^{(1)} L_{k}^{-\left(2 p-N d \alpha^{2}\right)} .
$$

Proof. The total number of pairs of cubes centered at lattice points, of radius $L_{j}$, inside $\mathbf{C}_{L_{j+1}}(\mathbf{0})$ (including separable ones) is, obviously, bounded by $\left|\mathbf{C}_{L_{j+1}}(\mathbf{0})\right|^{2} / 2$, so that we can apply the bound (2.14) and write

$$
\mathbb{P}\left\{\mathcal{S}_{j}\right\} \leq c\left(2 L_{j+1}+1\right)^{2 N d} L_{j-1}^{-2 p} \leq c^{\prime} L_{j-1}^{-2 p+2 N d \alpha^{2}}
$$

where $c$ and $c^{\prime}$ are constants depending on $d, N$ and $p$. Therefore,

$$
\Omega_{k}^{(\mathrm{bad})} \leq c^{\prime} L_{k}^{-2 p+2 N d \alpha^{2}} \sum_{i \geq 0}\left(\frac{L_{k+i}}{L_{k}}\right)^{-2 p+2 N d \alpha^{2}} .
$$

By assumptions, $2 p-N d \alpha>0$ and $L_{0} \geq 2$, so that the assertion of Lemma 7.1 follows from the inequality $L_{k}^{-1} L_{k+i} \geq 2^{\alpha^{k+i}-\alpha^{k}}$ (where $\alpha=3 / 2$ ).

7.2. Step 2. Centers of localization. Denote by $\boldsymbol{\Phi}_{l}=\boldsymbol{\Phi}_{l}(\omega)$ the normalized eigenfunctions of operator $\mathbf{H}^{(N)}(\omega)$, for $\omega \in \Omega_{1}$, for which the corresponding eigenvalues $E_{l}=E_{l}(I, \omega) \in I, l=1,2, \ldots$, with, say, $E_{1} \leq E_{2} \leq \ldots$. For each $l$ and $\omega \in \Omega_{1}$ we call a center of localization for function $\boldsymbol{\Phi}_{l}$ any point $\mathbf{z}_{l}=\mathbf{z}_{l}(\omega) \in \mathbb{Z}^{N d}$ such that

$$
\left\|\mathbf{1}_{\mathbf{C}_{1}\left(\mathbf{z}_{l}\right)} \boldsymbol{\Phi}_{l}\right\|=\max _{\mathbf{y} \in \mathbb{Z}^{N d}}\left\|\mathbf{1}_{\mathbf{C}_{1}(\mathbf{y})} \boldsymbol{\Phi}_{l}\right\| .
$$

Since $\boldsymbol{\Phi}_{l} \in L^{2}\left(\mathbb{R}^{N d}\right)$, such a center always exists. Moreover, owing to the normalization $\left\|\boldsymbol{\Phi}_{l}\right\|=1$, the number of centers of localization for a given $l$ must be finite. We will assume below that for each considered eigenfunction $\Phi_{l}$, precisely one center of localization $\mathrm{z}_{l}$ has been chosen (in a unique, but otherwise arbitrary way).

Lemma 7.2 (Cf. Sect. 3.4 from [20). Given $m>0$, there exists $k_{0}$ with the following property. Let $k \geq k_{0}$ and suppose that for some $l$ the center of localization $\mathbf{z}_{l}$ lies in a cube $\mathbf{C}_{L_{k}}(\mathbf{x})$. Then the cube $\mathbf{C}_{L_{k+1}}(\mathbf{x})$ is $\left(m, E_{l}\right)$-S.

7.3. Step 3. Annular regions. Fix $k_{0}$ from the Step 2 and set $\Omega_{k}^{\text {(good) }}=\Omega_{1} \backslash \Omega_{k}^{(\text {bad })}$.

Lemma 7.3. There exists $j_{0}=j_{0}(m, \alpha, d)$ large enough such that for $j \geq j_{0}, j \geq k$ and $\mathbf{z}_{l} \in \mathbf{C}_{L_{j}}(\mathbf{0})$

$$
\left\|\left(1-\mathbf{1}_{\mathbf{C}_{L_{j+2}}(\mathbf{0})}\right) \boldsymbol{\Phi}_{l}\right\| \leq \frac{1}{4}
$$


Proof. Let $k_{0}$ be the value from Lemma 7.2 Fix $j \geq k_{0}$ and divide the complement $\mathbb{Z}^{N d} \backslash \mathbf{C}_{L_{j+2}}(\mathbf{0})$ into annular regions

$$
\mathbf{M}_{i} \equiv \mathbf{M}_{i}(\mathbf{0}):=\mathbb{Z}^{N d} \bigcap\left(\mathbf{C}_{L_{i+1}}(\mathbf{0}) \backslash \mathbf{C}_{L_{i}}(\mathbf{0})\right), i \geq j+2 .
$$

Thus, we have

$$
\|\left(1-\mathbf{1}_{\mathbf{C}_{L_{j+2}}(\mathbf{0})} \boldsymbol{\Phi}_{l}\left\|^{2}=\sum_{i \geq j+2}\right\| \mathbf{1}_{\mathbf{M}_{i}} \boldsymbol{\Phi}_{l}\left\|^{2} \leq \sum_{i \geq j+2} \sum_{\mathbf{w} \in \mathbf{M}_{i}}\right\| \mathbf{1}_{\mathbf{C}_{L_{i-1}}(\mathbf{w})} \boldsymbol{\Phi}_{l} \|^{2} .\right.
$$

By Lemma 2.1 (cf. (2.9)), any cube $\mathbf{C}_{L_{i-1}}(\mathbf{w}) \subset \mathbf{M}_{i}, i \geq j+2$, is separable from $\mathbf{C}_{L_{i-1}}(\mathbf{0})$, since $|\mathbf{w}|>2(N+2)\left(L_{i-1}+\mathrm{r}_{1}\right)$. Furthermore, $\mathbf{x}_{i} \in \mathbf{C}_{L_{j}}(\mathbf{0}) \subset \mathbf{C}_{L_{i-2}}(\mathbf{0})$, and by Lemma 7.2 the cube $\mathbf{C}_{L_{i-1}}(\mathbf{0})$ must be $\left(m, E_{l}\right)$-S. The cubes $\mathbf{C}_{L_{i-1}}(\mathbf{w}), \mathbf{C}_{L_{i-1}}(\mathbf{0})$ are separable, so for any $\omega \in \Omega_{k}^{\text {(good) }}$, the cube $\mathbf{C}_{L_{i-1}}(\mathbf{w})$ is $\left(m, E_{l}\right)$-NS. Therefore,

$$
\left\|\mathbf{1}_{\mathbf{C}_{L_{i-1}}(\mathbf{w})} \boldsymbol{\Phi}_{l}\right\|^{2} \leq e^{-2 m L_{i}} .
$$

Since the cardinality of annulus $\mathbf{M}_{i}$ grows polynomially in $L_{i}$, the assertion follows.

\subsection{Step 4. A combinatorial bound.}

Lemma 7.4 (Cf. Sect. 3.4 from [20]). There exist $\zeta \in(0,+\infty)$ and $c^{(2)} \in(0,+\infty)$ such that $\forall$ ??? $k \geq 1$ ???, $\omega \in \Omega_{k}^{\text {(good) }}$ and $j \geq k$ :

$$
\operatorname{card}\left\{l: \mathbf{z}_{l} \in \mathbf{C}_{L_{j+1}}(\mathbf{0})\right\} \leq c^{(2)} L_{j+1}^{\alpha \zeta d} \text {. }
$$

\subsection{Step 5. The bound for 'good' samples of potential.}

Lemma 7.5. Given $L_{0}$ large enough and $m>0, \exists$ an integer $k_{1}=k_{1}\left(m, L_{0}\right)$ such that $\forall k \geq k_{1}, \omega \in \Omega_{k}^{\text {(good) }}$ and $\mathbf{x}$ from the annular region $\mathbf{M}_{k+1}$ (cf. Eqn (7.2)) the following bound holds true:

$$
\left\|\mathbf{1}_{\mathbf{C}_{L_{k}}(\mathbf{x})} \xi(H(\omega)) \mathbf{1}_{\mathbf{C}_{L_{k}}(\mathbf{0})}\right\| \leq e^{-m L_{k} / 2}\|\xi\|_{\infty}
$$

Here $\xi$ is a (measurable) bounded function $\mathbb{R} \rightarrow \mathbb{R}$.

Proof. It suffices to prove (7.4) in the particular case where $\|\xi\|_{\infty} \leq 1$, which we assume below. First, the operator norm $\left\|\mathbf{1}_{\mathbf{C}_{L_{k}}(\mathbf{x})} \xi(H(\omega)) \mathbf{1}_{\mathbf{C}_{L_{k}}(\mathbf{0})}\right\|$ in the LHS of (7.4) is upper-bounded by

$$
\begin{aligned}
\sum_{E_{l} \in I}\left|\xi\left(E_{l}\right)\right|\left\|\mathbf{1}_{\mathbf{C}_{L_{k}}(\mathbf{x})} \boldsymbol{\Phi}_{l}\right\|\left\|\mathbf{1}_{\mathbf{C}_{L_{k}}(\mathbf{0})} \boldsymbol{\Phi}_{l}\right\| \\
\leq \sum_{\substack{E_{l} \in I \\
\mathbf{z}_{l} \in \mathbf{C}_{L_{k+1}}}}\left\|\mathbf{1}_{\mathbf{C}_{L_{k}}(\mathbf{x})} \boldsymbol{\Phi}_{l}\right\|\left\|\mathbf{1}_{\mathbf{C}_{L_{k}}(\mathbf{0})} \boldsymbol{\Phi}_{l}\right\| \\
\quad+\sum_{j \geq k+1} \sum_{\substack{E_{l} \in I \\
\mathbf{z}_{l} \in \mathbf{M}_{j}}}\left\|\mathbf{1}_{\mathbf{C}_{L_{k}}(\mathbf{x})} \boldsymbol{\Phi}_{l}\right\|\left\|\mathbf{1}_{\mathbf{C}_{L_{k}}(\mathbf{0})} \boldsymbol{\Phi}_{l}\right\| .
\end{aligned}
$$

Observe that if point $\mathbf{x}$ lies in the annulus $\mathbf{M}_{k+1}=\mathbb{Z}^{N d} \cap\left(\mathbf{C}_{L_{k+2}}(\mathbf{0}) \backslash \mathbf{C}_{L_{k+1}}(\mathbf{0})\right)$, then $|\mathbf{x}|>2(N+2)\left(L_{k}+\mathrm{r}_{1}\right)$, and by Lemma 2.1 (cf. (2.9)), cubes $\mathbf{C}_{L_{k}}(\mathbf{x})$ and $\mathbf{C}_{L_{k}}(\mathbf{0})$ are separable. In turn, this implies that one of these cubes has to be $\left(m, E_{l}\right)$-non-singular, so we can write

$$
\sum_{\substack{E_{l} \in I \\ \mathbf{z}_{l} \in \mathbf{C}_{L_{k+1}}}}\left\|\mathbf{1}_{\mathbf{C}_{L_{k}}(\mathbf{x})} \boldsymbol{\Phi}_{l}\right\|\left\|\mathbf{1}_{\mathbf{C}_{L_{k}}(\mathbf{u})} \boldsymbol{\Phi}_{l}\right\| \leq c^{(2)} L_{k+1}^{\alpha \zeta d} e^{-m L_{k}},
$$


taking into account the upper bound for the number of points $\mathbf{z}_{l}$ obtained in Step 4.

If $\mathbf{z}_{l} \in \mathbf{M}_{j}$ with $j \geq k+1$, then by Lemma $7.2 \mathbf{C}_{L_{j-1}}\left(\mathbf{z}_{l}\right)$ is $\left(m, E_{l}\right)$-S. Since $\left|\mathbf{z}_{l}\right|>2(N+2)\left(L_{j-1}+\mathrm{r}_{1}\right)$, the cube $\mathbf{C}_{L_{j-1}}(\mathbf{0})$ must be $\left(m, E_{l}\right)$-NS. Consequently, we have the upper-bounds for the norms:

$$
\left\|\mathbf{1}_{\mathbf{C}_{L_{k}}(\mathbf{0})} \boldsymbol{\Phi}_{l}\right\| \leq\left\|\mathbf{1}_{\mathbf{C}_{L_{j-1}}(\mathbf{0})} \boldsymbol{\Phi}_{l}\right\| \leq c^{(3)} e^{-m L_{j-1}}
$$

where $c^{(3)} \in(0,+\infty)$ is a constant, $c^{(3)}=c^{(3)}(d, N)$. Using again Lemma 7.4] we see that for $k \geq k_{1}$,

$$
\sum_{j \geq k+1} \sum_{\mathbf{z}_{l} \in \mathbf{M}_{j}}\left\|\mathbf{1}_{\mathbf{C}_{L_{k}}(\mathbf{x})} \boldsymbol{\Phi}_{l}\right\|\left\|\mathbf{1}_{\mathbf{C}_{L_{k}}(\mathbf{0})} \boldsymbol{\Phi}_{l}\right\| \leq c^{(3)} \sum_{j \geq k+1} e^{-m L_{j-1}} L_{j}^{\alpha \zeta d} \leq \frac{1}{2} e^{-m L_{k} / 2} .
$$

Combined with (7.5), this estimate gives the assertion of Lemma 7.5

\subsection{Step 6. The overall bound.}

Lemma 7.6. $\exists$ a constant $c^{(4)}=c^{(4)}(d, N, p) \in(0,+\infty)$ with the following property. Let $k_{1}$ be as in Step 5. Then for $k \geq k_{1}$ and $\mathbf{x} \in \mathbf{M}_{k+1}$, we have:

$$
\mathbb{E}\left[\left\|\mathbf{1}_{\mathbf{C}_{L_{k}}(\mathbf{x})} \xi(H(\omega)) \mathbf{1}_{\mathbf{C}_{L_{k}}(\mathbf{0})}\right\|\right] \leq c^{(4)}\|\xi\|_{\infty} L_{k}^{-2 p+2 N d \alpha^{2}} .
$$

Proof. For $\omega \in \Omega_{k}^{\text {(bad) }}$ we can estimate the norm by $\|\xi\|_{\infty} \leq 1$, while for $\omega \in \Omega_{k}^{\text {(good) }}$ we apply Lemma 7.5 Using Lemma 7.1] we see that the above expectation is bounded by

$$
\left(\mathbb{P}\left\{\Omega_{k}^{(\mathrm{bad})}\right\}+e^{-m L_{k} / 2} \mathbb{P}\left\{\Omega_{k}^{(\text {good })}\right\}\right) \leq C(\alpha, d, p) L_{k}^{-2 p+2 N d \alpha^{2}}(1+o(1))
$$

7.7. Step 7. Conclusion. Fix a compact $\mathbb{K} \subset \mathbb{R}^{N d}$ and find $k \geq k_{1}$ such that $\mathbb{K} \subset \mathbf{C}_{L_{k-1}}(\mathbf{0})$. Then

$$
\begin{aligned}
& \mathbb{E}\left[\left\|\mathbf{X}^{s} \xi(H(\omega)) \mathbf{1}_{\mathbb{K}}\right\|\right] \leq c_{d} L_{k}^{s}+\sum_{j \geq k} \mathbb{E}\left[\left\|\mathbf{X}^{s} \mathbf{1}_{\mathbf{M}_{j}} \xi(H(\omega)) \mathbf{1}_{\mathbb{K}}\right\|\right] \\
& \leq c(k)+\sum_{j \geq k} c_{d} L_{j+1}^{s} \sum_{\mathbf{w} \in \mathbf{M}_{j}} \mathbb{E}\left[\left\|\mathbf{1}_{\mathbf{C}_{L_{k}}(\mathbf{w})} \xi(H(\omega)) \mathbf{1}_{\mathbf{C}_{L_{k}}(\mathbf{0})}\right\|\right] \\
& \leq C\left[1+\sum_{j \geq k} L_{j}^{\alpha s} L_{j}^{N d \alpha} L_{j}^{-2 p+2 N d \alpha^{2}}\right]<\infty,
\end{aligned}
$$

since $2 p-3 N d \alpha^{2}-\alpha s>0$ by assumption (7.1), and $L_{j}=\left(L_{0}\right)^{\alpha^{j}}$ grow fast enough.

This completes the proof of dynamical localization. ??? Vitya: at what point in Section 7 do we have to assume that $I$ is of the form $I^{0}(\eta)$ ???

\section{Appendix. Geometric separability.}

In physical terms, the condition of separability can be elucidated as follows: let cube $\mathbf{C}_{L}^{(n)}(\mathbf{x})$ be $\mathcal{J}$-separable from $\mathbf{C}_{L}^{(n)}(\mathbf{y})$ and consider two quantum $n$-particle systems, in $\mathbf{C}_{L}^{(n)}(\mathbf{x})$ and $\mathbf{C}_{L}^{(n)}(\mathbf{y})$ (i.e., with Hamiltonians $\mathbf{H}_{\mathbf{C}_{L}^{(n)}(\mathbf{x})}$ and $\mathbf{H}_{\mathbf{C}_{L}^{(n)}(\mathbf{y})}$ ). Then the first system contains a 'detached' subsystem, formed by particles with labels from $\mathcal{J}$, with the following property. $\forall \mathbf{u}=\left(u_{1}, \ldots, u_{n}\right) \in \mathbf{C}_{L}^{(n)}(\mathbf{x})$ and $\mathbf{v}=\left(v_{1}, \ldots, v_{n}\right) \in \mathbf{C}_{L}^{(n)}(\mathbf{y})$, the collection of random variables $\mathrm{V}_{s}$ from the random field $V$ contributing to the 
external potential sum $\sum_{j \in \mathcal{J}} V\left(x_{j} ; \omega\right)$ is disjoint from similarly defined collections, for sums $\sum_{j \notin \mathcal{J}} V\left(x_{j} ; \omega\right)$ and $\sum_{1 \leq j \leq n} V\left(x_{j}^{\prime} ; \omega\right)$. This implies independence of sum $\sum_{j \in \mathcal{J}} V\left(x_{j} ; \omega\right)$ and the pair of sums $\sum_{j \notin \mathcal{J}} V\left(x_{j} ; \omega\right)$ and $\sum_{1 \leq j \leq n} V\left(x_{j}^{\prime} ; \omega\right)$ and provides enough 'randomness' to produce satisfactory estimates.

Proof of Corollary 2.1. Assume that the assertion is wrong and consider $\kappa(n)+1$ cubes $\mathbf{C}_{L}\left(\mathbf{y}_{j}\right) \subset \boldsymbol{A}_{2 j-1}(\mathbf{x}), 1 \leq j \leq \kappa(n)+1$, which are not separable from $\mathbf{C}_{L}(\mathbf{x})$. Since

$$
\operatorname{dist}\left(\mathbf{C}_{L}\left(\mathbf{y}_{j}\right), \mathbf{C}_{L}\left(\mathbf{y}_{j+1}\right)\right) \geq \operatorname{dist}\left(\boldsymbol{A}_{j}(\mathbf{x}), \boldsymbol{A}_{j+1}(\mathbf{x})\right)-2\left(L+\mathrm{r}_{1}\right)>4 n\left(L+\mathrm{r}_{1}\right),
$$

these $\kappa(n)+1$ cubes cannot be enclosed in $\kappa(n)$ cubes of radius $2 n\left(L+\mathrm{r}_{1}\right)$, in contradiction to the first assertion of Lemma 2.1

Proof of Lemma 5.1. If $\mathbf{C}_{L}\left(\mathbf{u}^{\prime}\right)$ is FI, then there exists a permutation $\sigma$ of order $N$ such that, for all $j=1, \ldots, N-1,\left|u_{j}^{\prime}-u_{j+1}^{\prime}\right| \leq \mathrm{r}_{0}$. Otherwise, the set $\left\{u_{j}^{\prime}\right\}_{1 \leq j \leq N} \subset \mathbb{Z}^{d}$ could be decomposed into two or more non-interacting subsets. Therefore,

$$
\operatorname{diam}\left\{u_{j}^{\prime}\right\}_{1 \leq j \leq n} \leq(N-1) \mathrm{r}_{0} ; \text { similarly, } \operatorname{diam}\left\{u_{j}^{\prime \prime}\right\}_{1 \leq j \leq n} \leq(N-1) \mathrm{r}_{0} .
$$

Further, suppose that for some $i, j \in\{1, \ldots, n\}$, we have

$$
\Pi_{i} \mathbf{C}_{L+\mathrm{r}_{1}}\left(\mathbf{u}^{\prime}\right) \cap \Pi_{j} \mathbf{C}_{L+\mathrm{r}_{1}}\left(\mathbf{u}^{\prime \prime}\right) \neq \varnothing .
$$

Then $\left|u_{i}^{\prime}-u_{j}^{\prime \prime}\right| \leq 2\left(L+\mathrm{r}_{1}\right)$, and, therefore, for any $k=1, \ldots, n$

$$
\begin{aligned}
\left|u_{k}^{\prime}-u_{k}^{\prime \prime}\right| & \leq\left|u_{k}^{\prime}-u_{i}^{\prime}\right|+\left|u_{i}^{\prime}-u_{j}^{\prime \prime}\right|+\left|u_{j}^{\prime \prime}-u_{k}^{\prime \prime}\right| \\
& \leq(N-1) \mathrm{r}_{0}+2\left(L+\mathrm{r}_{1}\right)+(N-1) \mathrm{r}_{0} \leq 2 N\left(L+\mathrm{r}_{1}\right) .
\end{aligned}
$$

This is incompatible with the inequality $\operatorname{dist}\left(\mathbf{C}_{L}\left(\mathbf{u}^{\prime}\right), \mathbf{C}_{L}\left(\mathbf{u}^{\prime \prime}\right)\right)>2 n N\left(L+\mathrm{r}_{1}\right)$, since in the latter case there must exist some $k$ such that $\left|u_{k}^{\prime}-u_{k}^{\prime \prime}\right|>2\left(L+\mathrm{r}_{1}\right)$.

\section{ACKNOWLEDGEMENTS}

The authors thank Peter Stollmann for valuable discussions which contributed into technical aspects of the material of this paper. Besides, Peter was a valuable collaborator in [6] and [7. It is also a pleasure to thank Francois Germinet for fruitful exchange in Summer, 2007. VC thanks The Isaac Newton Institute (INI) and Department of Pure Mathematics and Mathematical Statistics, University of Cambridge, for hospitality during visits in 2003, 2004, 2007 and 2008. YS thanks IHES, Bures-sur-Yvette, and STP, Dublin Institute for Advanced Studies, for hospitality during visits in 20072008. YS thanks the Departments of Mathematics of Penn State University and of UC Davis, for hospitality during Visiting Professorships in Winter of 2008. YS thanks the Department of Physics, Princeton University and the Department of Mathematics of UC Irvine, for hospitality during visits in the Spring of 2008.

\section{REFERENCES}

[1] M. Aizenman and B. Simon, Brownian motion and Harnack's inequality for Schrödinger operators, Comm. Pure Appl. Math 35 (1982), 209-273. $\uparrow 5$

[2] M. Aizenman, A. Elgart, S. Naboko, G. Stoltz, and J.H. Schenker, Moment analysis for localization in random Schrd̈inger operators, Invent. Mathem. 163 (2006), 343-413. $\uparrow 2$

[3] M. Aizenman and S. Warzel, Localization bounds for multiparticle systems, Comm. Math. Phys. 290 (2009), no. 3, 903-934. $\uparrow 2$

[4] - Complete dynamical localization in disordered quantum multi-particle systems, 2009, arXiv:math-ph/0909:5432 (2009). $\uparrow 2$ 
[5] A. Boutet de Monvel, V. Chulaevsky, P. Stollmann, and Y. Suhov, Wegner-type bounds for a multi-particle continuous Anderson model with an alloy-type external potential, J. Stat. Phys. 138 (2009), no. 4-5, 553-566. $\uparrow 7$

[6] , Anderson localization in a two-particle model with an alloy-type external potential, arXiv:0907.1459 (2009). $\uparrow 1,21$

[7] - Anderson localization for a multi-particle model with an alloy-type external potential, arXiv:1004.1300 (2010). $\uparrow 1,5,6,7,9,12,15,21$

[8] V. Chulaevsky, A remark on charge transfer processes in multi-particle systems, 2010, arXiv:math-ph/1005.3387. $\uparrow 6$

[9] V. Chulaevsky, A. Boutet de Monvel, and Y. Suhov, Multi-particle dynamical localization in a continuous Anderson model with an alloy-type potential, arXiv:1007.3815 (2010). $\uparrow 1,5,6$

[10] H.L. Cycon, R.G. Froese, W. Kirsch, and B. Simon, Schrödinger Operators with Applications to Quantum Mechanics and Global Geometry, Texts and Monographs in Physics, Springer, Berlin, Heidelberg, New York, 1987. $\uparrow 5$

[11] J.-M. Combes and P.D. Hislop, Localization for some continuous, random Hamiltonian in $d$ dimension, J. Funct. Anal. 124 (1994), 149-180. $\uparrow 2$

[12] V. Chulaevsky and Y. Suhov, Wegner bounds for a two-particle tight binding model, Comm. Math. Phys. 283 (2008), no. 2, 479-489. $\uparrow 2,6$

[13] (2009), no. 2, 701-723. $\uparrow 2,6,9$

[14] , Multi-particle Anderson localisation: induction on the number of particles, Math. Phys. Anal. Geom. 12 (2009), no. 2, 117-139. $\uparrow 2,6,9$

[15] H. von Dreifus and A. Klein, A new proof of localization in the Anderson tight binding model, Comm. Math. Phys. 124 (1989), no. 2, 285-299. $\uparrow 9$

[16] D. Damanik and P. Stollmann, Multi-scale analysis implies strong dynamical localization, Geom. Funct. Anal. 11 (2001), no. 1, 11-29. $\uparrow 17$

[17] F. Germinet and S. De Bièvre, Dynamical localization for discrete and continuous random Schrödinger operators, Comm. Math. Phys. 194 (1998), 323-341. $\uparrow 17$

[18] F. Klopp, Localization for continuous random Schrodinger operators, Vol. 167, 1995. $\uparrow 2$

[19] B. Simon, Schrödinger semigroups, Bull. Am. Math. Soc. 7 (1982), 447-526. $\uparrow 5$

[20] P. Stollmann, Caught by Disorder. Bound States in Random Media, Progress in Mathematical Physics, vol. 20, Birkhäuser Boston Inc., Boston, MA, 2001. $\uparrow 6,9,17,18,19$

${ }^{1}$ Département de Mathématiques, Université de Reims, Moulin de la Housse, B.P. 1039,, 51687 Reims Cedex 2, France, E-mail: victor.tchoulaevski@univ-Reims.Fr

${ }^{2}$ Institut de Mathématiques de Jussieu, Université Paris Diderot Paris 7, 175 Rue du Chevaleret, 75013 Paris, France, E-mail: aboutet@math.Jussieu.fr

${ }^{3}$ Statistical Laboratory, DPMms, University of Cambridge, Wilberforce Road, CamBIDGe CB3 0WB, UK, E-MaIL: Y.M.Suhov@Statslab.CAM.AC.uK 